\title{
Huangbai Liniment Accelerated Wound Healing by Activating Nrf2 Signaling in Diabetes
}

\author{
Jingjing Zhang $\mathbb{D}^{1},{ }^{1}$ Rui Zhou, ${ }^{1,2}$ Changpei Xiang, ${ }^{2}$ Qiang Jia $\mathbb{D}^{3},{ }^{3}$ Hongwei Wu $\mathbb{D}^{1}$ \\ and Hongjun Yang ${ }^{1,2}$ \\ ${ }^{1}$ Institute of Chinese Materia Medica, China Academy of Chinese Medical Sciences, Beijing 100700, China \\ ${ }^{2}$ College of traditional Chinese Medicine, Yunnan University of Traditional Chinese Medicine, Kunming 650500, China \\ ${ }^{3}$ School of Pharmacy, Shandong University of Traditional Chinese Medicine, Jinan 250355, China
}

Correspondence should be addressed to Hongjun Yang; hjyang@icmm.ac.cn

Received 17 May 2019; Revised 23 December 2019; Accepted 19 February 2020; Published 26 May 2020

Guest Editor: Reggiani Vilela Gonçalves

Copyright ( 2020 Jingjing Zhang et al. This is an open access article distributed under the Creative Commons Attribution License, which permits unrestricted use, distribution, and reproduction in any medium, provided the original work is properly cited.

\begin{abstract}
As a serious complication of diabetes, nonhealing skin ulcer leads to high mortality and disability in diabetic patients. However, limited therapy is available in managing diabetic wounds. In this study, RNA-seq technology was used to systematically investigate the effect of Huangbai (HB) liniment, a traditional Chinese medicine, on the streptozotocin- (STZ-) induced diabetic wound. HB liniment significantly accelerated the wound closure and enhanced the generation of extracellular matrix in diabetic rats, and oxidative stress was identified to play a vital role in HB-mediated wound healing. Importantly, HB liniment activated nuclear factor erythroid-derived 2-like 2 (Nrf2) and its downstream antioxidant genes (e.g., genes involved in glutathione system, thioredoxin system, and GAPDH generation as well as other antioxidant genes), which inhibited oxidative damage and apoptosis. By associating drug targets of $\mathrm{HB}$ liniment with Nrf2 and its downstream genes, 54 components in HB liniment were screened out, and the majority was from Cortex Phellodendri and Forsythia suspensa. Additionally, HB liniment enhanced TGF- $\beta 1$ and reduced MMP9 level, accelerating wound healing in diabetes. The in vitro experiment showed HB facilitated cell proliferation and inhibited oxidative damage in high glucose-induced HaCaT cells. Our findings provided the experimental evidence for the treatment of diabetic wound with $\mathrm{HB}$, clarified the potential mechanism of $\mathrm{HB}$, and improved our understanding of diabetic wound healing.
\end{abstract}

\section{Introduction}

Chronic nonhealing wound is a serious diabetic complication, which leads to severe morbidity and mortality in diabetic population and brings a huge social and economic burden to the word $[1,2]$. In the United States alone, it costs as high as $\$ 13$ billion to treat diabetic wounds every year. In contrast to the typical sequential emergence of biological process of coagulation, inflammation, proliferation, and remodeling in normal tissue, the normal progression of wound healing is disturbed and delayed in diabetes, resulting in long-term of wound nonunion [3, 4]. Conventional therapies are only effective in the management of diabetic wounds to certain degree, whereas a large number of diabetic wounds still persist, deteriorate, and result in amputation [5]. Notably, a reduced efficiency in diabetic wound healing is usually accompanied with decreased blood supply, delayed extracellular matrix turnover, reduced wound contraction, repeated infections, and chronic inflammation, which hinders wound healing $[4,6]$. Thus, it is urgent to develop an effective therapy to treat diabetic wounds.

Oxidative stress plays a vital role in halting the progression of diabetic wound healing $[7,8]$. The oxidative stress in diabetic wounds is characterized by a marked elevation in reactive oxygen species (ROS) levels, as a result of increasing ROS generating pathway and decreasing ROS removing defenses [9]. Overproduced ROS in diabetes damages various macromolecules such as lipids, proteins, and DNA double strands and finally impairs wound healing. Proper oxidative stress has been proved to benefit extracellular matrix (ECM) generation, whereas high ROS levels hinder normal synthesis of ECM and seriously damage existing ECM 
$[10,11]$. For example, $\mathrm{H}_{2} \mathrm{O}_{2}$ can disrupt tissue growth, especially collagen production, thus preventing wound closure [12]. In addition, TGF- $\beta 1$ signaling is a vital signaling pathway for ECM production and can be interfered by the overproduced ROS. Importantly, high ROS levels promote the generation of matrix metalloproteinases (MMPs), which in turn affect ECM remodeling [13]. Therefore, targeting oxidative stress may be a potential effective therapy for diabetic wound healing.

An increasing number of researchers have demonstrated Traditional Chinese medicine (TCM) is indispensable in the treatment of various disease presentations [14, 15]. Abundant and robust evidences accumulated over time that TCM is effective in its use for diabetic complications [16]. Huangbai liniment $(\mathrm{HB})$ is a standardized medicinal product clinically prescribed for use in wound management and is composed of Cortex Phellodendri, Forsythia suspensa, Lonicera japonica Thunb., Taraxacum mongolicum Hand.Mazz, and Scolopendridae [17]. Therapeutic benefits of HB liniment have been observed in wound management, including nonhealing diabetic ulcer $[18,19]$. These study demonstrated that $\mathrm{HB}$ liniment could reduce AGEs and inflammatory factors such as IL- $1 \beta$ while increasing the secretion of growth factor [20]. However, the mechanism of $\mathrm{HB}$ in facilitating diabetic wound healing remains unclear. In this study, the effect of $\mathrm{HB}$ on diabetic wound healing was evaluated in STZ-induced iabetic wounds and high glucose-induced cell model, and the mechanism was systematically investigated by using RNA-seq technology.

\section{Materials and Methods}

2.1. Materials. Streptozotocin (STZ, SigmaS0130) and 4',6-diamidino-2-phenylindole (DAPI) were purchased from Sigma-Aldrich. Huangbai liniment (batch number: 18010111) was kindly provided by Shandong Hanfang Pharmaceutical Co., Ltd (Chinese medicine character: Z10950097). Recombinant human epidermal growth factor derivative for external use (rhEGF) was purchased from Shenzhen Huashengyuan Gene Engineering Development Co., Ltd. The antibodies used in this research were as follows: Ki67 (ab92742), 8-OHdG (sc-66036), Nrf2 (ab137550), NQO1 (ab28947), cleaved caspase 3 (CST, 9664S), TGF- $\beta 1$ (ab92486), goat anti-mouse IgG (H+L) (HRP Jackson, 115-035-003), and goat anti-rabbit IgG $(\mathrm{H}+\mathrm{L})$ (Jackson, 111-035-003). The malondialdehyde (MDA, A003) was purchased from Nanjing Jiancheng Bioengineering Institute. The ELISA kits of MMP9 (DY-0075) and 8-OHdG (DY-0223) were obtained from Deyi Testing Co., Ltd. Cell Counting Kit-8 (CCK-8) kits were obtained from the Dongren Chemical Technology Co., Ltd. The malondialdehyde ROS (S0033) and GSH (A119) were purchased from Beyotime Biotechnology and Nanjing Jiancheng Bioengineering Institute, respectively.

2.2. In Vivo Animal Study. Male Sprague-Dawley rats (170-200 g) were purchased from the Peking University Health Science Center Experimental Animal Center, Beijing, China ((certificate no. SCXK (Jing) 2009-0017)). All proce- dures were carried out according to the rules of the Committee on Animal Care and Use published by the Institute of Chinese Materia Medica, China Academy of Chinese Medical Sciences. Rats were housed in a 12-h light/dark cycle facility with a controlled temperature and kept with free access to water and food. Streptozotocin (STZ, $60 \mathrm{mg} / \mathrm{kg}$, i.p.) in sodium citrate buffer ( $\mathrm{pH} 4.5$ ) was used to generate diabetic model according to previous reports [21]. The fasting glucose levels (FGLs) was evaluated 1 and 2 weeks following STZ injection. Rats with FGL more than $16.7 \mathrm{mmol}$ were included for the following experiments. The control animals received the same volume of sodium citrate buffer ( $\mathrm{pH} 4.5$ ) instead. At two weeks after STZ induction, the rats were anesthetized with sodium pentobarbital $(50 \mathrm{mg} / \mathrm{kg}$, intraperitoneal injection, i.p.) and then subjected to full-thickness wounds. Two wounds with diameter of $2 \mathrm{~cm}$ on the back of rats were made. The rats were randomly allocated to different groups to receive either physiological saline, low dose of Huangbai liniment (STZ+HB-L, equal volume of $\mathrm{HB}+$ equal volume of physiological saline, $2 \mathrm{~mL}$ /day, topical), high dose of Huangbai liniment STZ+HB-H (original solution, $2 \mathrm{~mL} /$ day, topical), or rhEGF (4000 IU/10* $10 \mathrm{~cm}^{2}$, topical) until study end. On the first three days after wound surgery, the wound was covered with double layers of sterile gauze to reduce external irritation and replaced daily. The wounds were photographed at 1, 6, 9, and 13 days after surgical wounds were made. The wound closure photos were analyzed using ImageJ by calculating the percentage of the wound area from the original wound area. After 13 days, skin tissues were collected, half of which were paraffin-embedded for histology, and the other half were used for western blotting, ELISA, and other related experiments.

2.3. In Vitro Cell Experiments. Human immortalized keratinocytes $(\mathrm{HaCaT})$ purchased from National Infrastructure of Cell Line Resource was used for our in vitro experiment. Briefly, HaCaT cells were cultured with culture medium which contained DMEM (Gibco, USA) with D-glucose $(1 \mathrm{~g} / \mathrm{L}, \mathrm{LG})$, fetal bovine serum $(10 \% \mathrm{v} / \mathrm{v}, \mathrm{FBS})$, and penicillin/streptomycin $(100 \mu \mathrm{g} / \mathrm{mL})$ at $37^{\circ} \mathrm{C}$, and stimulation of $\mathrm{HaCaT}$ cells with culture medium containing $4.5 \mathrm{~g} / \mathrm{L}$ D-glucose DMEM (HG) for 48 hours was used for chronic hyperglycemic cell model. Povidone iodine and quercetin were purchased from Hubei Ketian Pharmaceutical Co., Ltd and National Institutes for Food and Drug Control, respectively. First, $\mathrm{HaCaT}$ cells were treated with various concentrations of HB, quercetin, and povidone iodine, respectively, for 48 hours to search the safe concentration. To evaluate the therapeutic effect, cells were treated with HG medium and drugs such as $\mathrm{HB}$ (1/50 and 1/5000, dilution), quercetin $(1.5625$ and $6.25 \mu \mathrm{M})$, and povidone iodine $(0.00001 \%$ and $0.001 \%, \mathrm{~m} / \mathrm{v}$ ) at the same time for 48 hours before detection. CCK-8 was used for detecting cell viability with a microplate reader (Molecular Devices, USA).

2.4. Measurement of Malondialdehyde, GSH, ROS, 8-OHdG, and MMP9. Malondialdehyde (MDA) was detected according to the manufacture instrument, using Visible Spectrophotometer 721G (Shanghai Precision Scientific 
Instruments Co., Ltd.). And GSH and ROS levels were also performed according to their instruction using a microplate reader (Molecular Devices, USA). The protein extract from the skin tissue was used for the detection of MMP9 and 8-OHdG using commercially available kits. Briefly, plates were immobilized with specific antibody (MMP9 or $8-\mathrm{OHdG}$ ) and incubated with protein samples, followed by a secondary antibody conjugated with horseradish peroxidase. The measurement was performed on Beijing DNM-9602G Enzyme Marker Analyzer.

2.5. Western Blotting. The skin tissue was harvested, and protein extraction was performed according to the instruction of Beyotime Protein Assay Kit (P0013, Nanjing, China). After separated on a $10 \%$ SDS-PAGE (P0014, China), the protein samples were transferred to a polyvinylidene fluoride membrane (Millipore, IPVH00010), which was blocked with bovine serum albumin (BSA). After that, the primary antibody specific for Nrf2 (ab137550) or NQO1 (ab28947) was added to the membranes and incubated for $24 \mathrm{~h}$ at $4^{\circ} \mathrm{C}$. The second antibody goat anti-rabbit IgG $(\mathrm{H}+\mathrm{L})$ (Jackson, 111-035-003) was loaded, and the signal of the proteins was detected by scanning densitometry.

2.6. Histology and Immunofluorescence Staining. The paraffin tissue section slides with thickness of $5 \mu \mathrm{m}$ were used for histology and immunofluorescence staining. After deparaffinized, the slides were stained with hematoxylin and eosin (HE) and Masson's trichrome stain following their instructions, respectively. For immunofluorescence (IF) staining, the sections were blocked by $5 \%(\mathrm{~m} / \mathrm{v}) \mathrm{BSA}$, after they were permeabilized with $0.5 \%$ Triton X-100. Primary antibody was loaded and kept at $4^{\circ} \mathrm{C}$ overnight. Then Alexa Fluor 488- and Alexa Fluor 647-conjugated secondary antibodies were loaded at room temperature for $1 \mathrm{~h}$. The nuclei were stained with $4^{\prime}, 6$-diamidino-2-phenylindole (DAPI). The images were visualized using LSM-880 confocal microscope (Carl Zeiss, Oberkochen, Germany). And the real-time collagen growth evaluation was conducted using twophoton microscope (Olympus FV1000) at 6 and 13 days after wound generation.

2.7. Investigation and Analysis of Differentially Expressed Genes Identified by RNA-Seq Technology. The skin tissue was harvested, and the RNA was separated by using Total TRIzol Reagent (Cat\#15596-018, Life Technologies, USA) following its instructions. RNA integrity was quantified with the RNA Nano 6000 Assay Kit using Bioanalyzer 2100 system (Agilent Technologies, CA, USA). The RNA $(1 \mu \mathrm{g})$ of each sample was used for the following library construction. The RNA sequencing was performed according to the previous research [15]. Briefly, NEBNext ${ }^{\circledR}$ Ultra $^{\text {TM }}$ RNA Library Prep Kit was applied to produce the sequencing libraries for Illumina ${ }^{\circledR}$ (NEB, USA). Firstly, purification of mRNA was carried out by using poly-T oligo-grafted magnetic beads before RNA fragmentation was generated. After that, the first strand cDNA was synthesized by using M-MuLV Reverse Transcriptase(RNase $\mathrm{H}-$ ) and random hexamer primer. And the second strand cDNA was synthesized with RNase
$\mathrm{H}$ and DNA polymerase I. The enrichment of cDNA template by PCR was carried out after knot of the adapter and adenylation at the $3^{\prime}$ end in the DNA fragment. After purification of PCR results, the library quality was evaluated, and the clustering of samples was performed on TruSeq PE150 Cluster Kit v3-cBot-HS (Illumia) with a cBot Cluster Generation System. Finally, the sequence of the library was carried out by using an Illumina HiSeq 4000 platform with the generation of $150 \mathrm{bp}$ paired-end reads. Novogene Bioinformatics Technology Co., Ltd (Beijing, China) helped to do the sequencing experiment.

Rat reference genome (ensemble release 91) was applied for the transcript assembly and quantification. The numbers of reads were calculated by mapping to every gene with HTseq v0.6.1. Gene expression was obtained by evaluating the fragment number of each kilobase of transcript sequence normalized to every million base pairs which were sequenced and expressed as FPKM. Differential expression of genes was calculated with EdgeR software. The raw data related to HB-mediated protection against STZ-induced diabetic wound was uploaded into https://www.ncbi.nlm.nih.gov/ sra/PRJNA532974 (SRP193129). The differentially expressed genes identified by RNA-seq were analyzed through ClueGO. And the network of "components-drug targetsdifferentially expressed genes" was constructed with Cytoscape v3.4.0. [22]. Briefly, the drug targets of HB liniment were obtained through BATMAN-TCM, and the downstream differentially expressed genes of Nrf2 and $\mathrm{Nfe} 2 \mathrm{l} 2$ were associated with the drug targets by STRING to screen out related drug targets, and the network was constructed through Cytoscape v3.4.0.

2.8. Statistical Analysis. The data was analyzed using one-way ANOVA with Tukey post hoc test through SPSS software, and the data was presented as means $\pm S D$ (standard deviations). ${ }^{\#} P<0.05$ compared with the Con or LG group, ${ }^{*} P<0.05$ compared with the STZ or HG group was considered as statistical significance.

\section{Results}

3.1. HB Liniment Accelerated Wound Closure in STZ-Induced Diabetic Rats. The effect of HB liniment on diabetic wound healing was investigated through the evaluation of the wound closure and histology staining. As indicated in Figures 1(b) and $1(\mathrm{c})$, the wound closure in the STZ group was slower than those of the control group. Both low- and high-dose HB liniments increased the wound closure to the level of the STZ+rhEGF group in STZ-induced diabetic wounds, but it cannot promote wound healing of nondiabetic rats until at 13 days after operation. Additionally, HB liniment and rhEGF treated rats demonstrated a stronger staining than that of the STZ group as indicated by HE staining and Masson staining (Figure 1(d)), indicating improved ECM synthesis and collagen production by $\mathrm{HB}$ and rhEGF treatments. These results demonstrated that $\mathrm{HB}$ treatment promoted wound healing in STZ-induced diabetic rats. 
HB treatment

(Original liquid, $2 \mathrm{ml}$, qd)

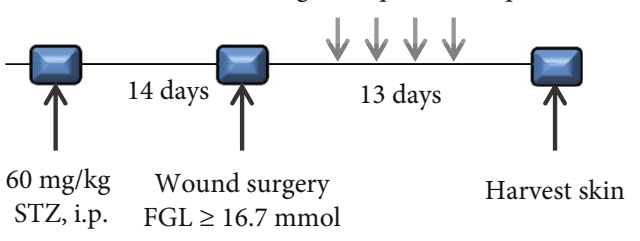

(a)

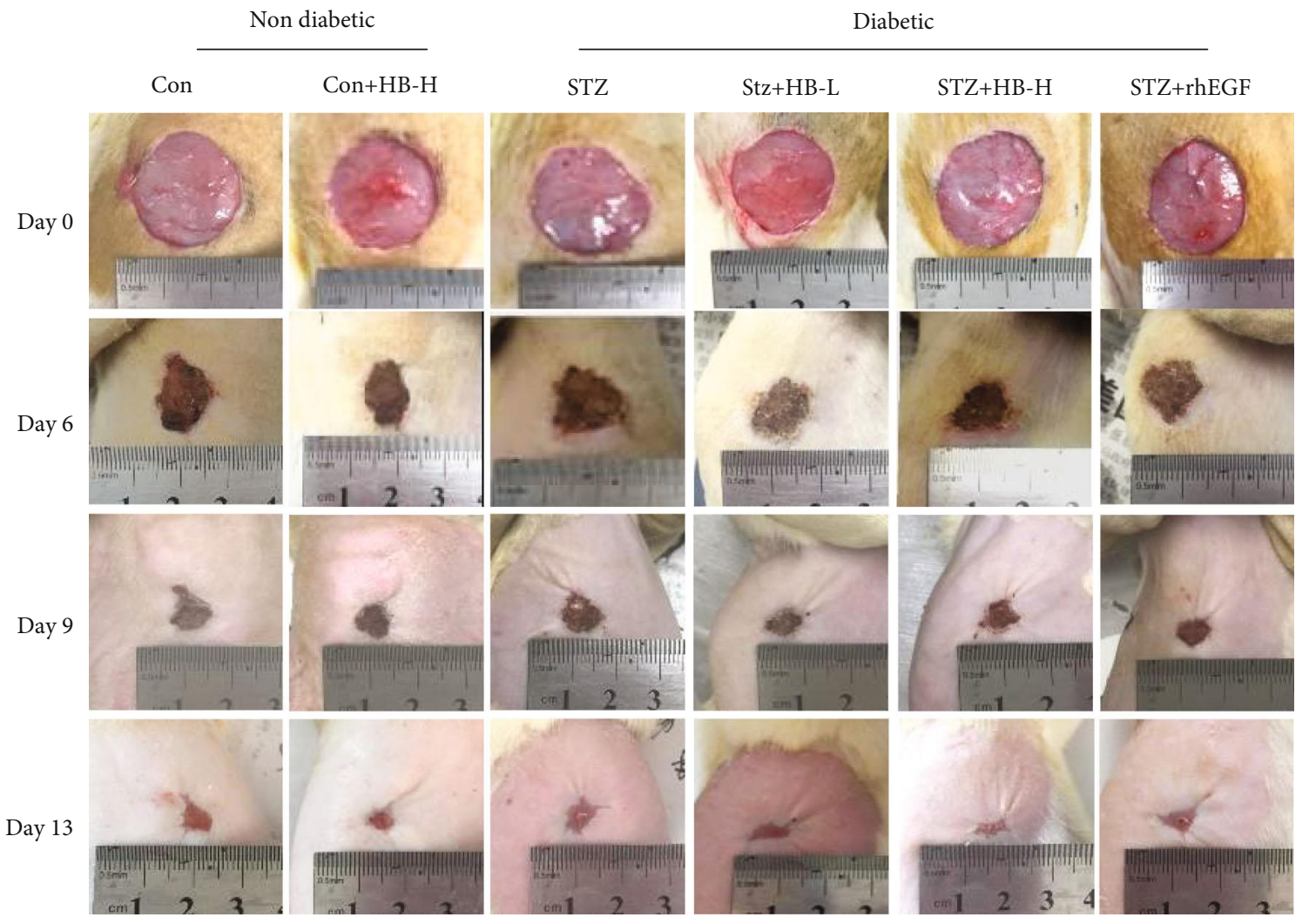

(b)

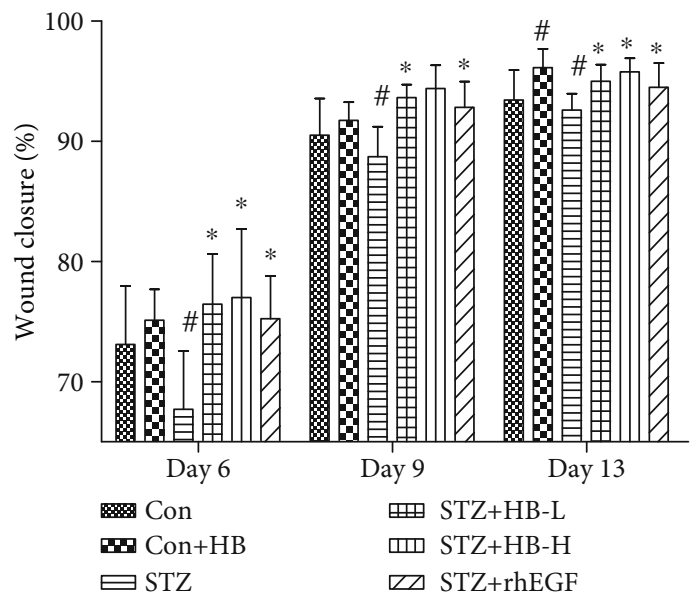

(c)

Figure 1: Continued. 


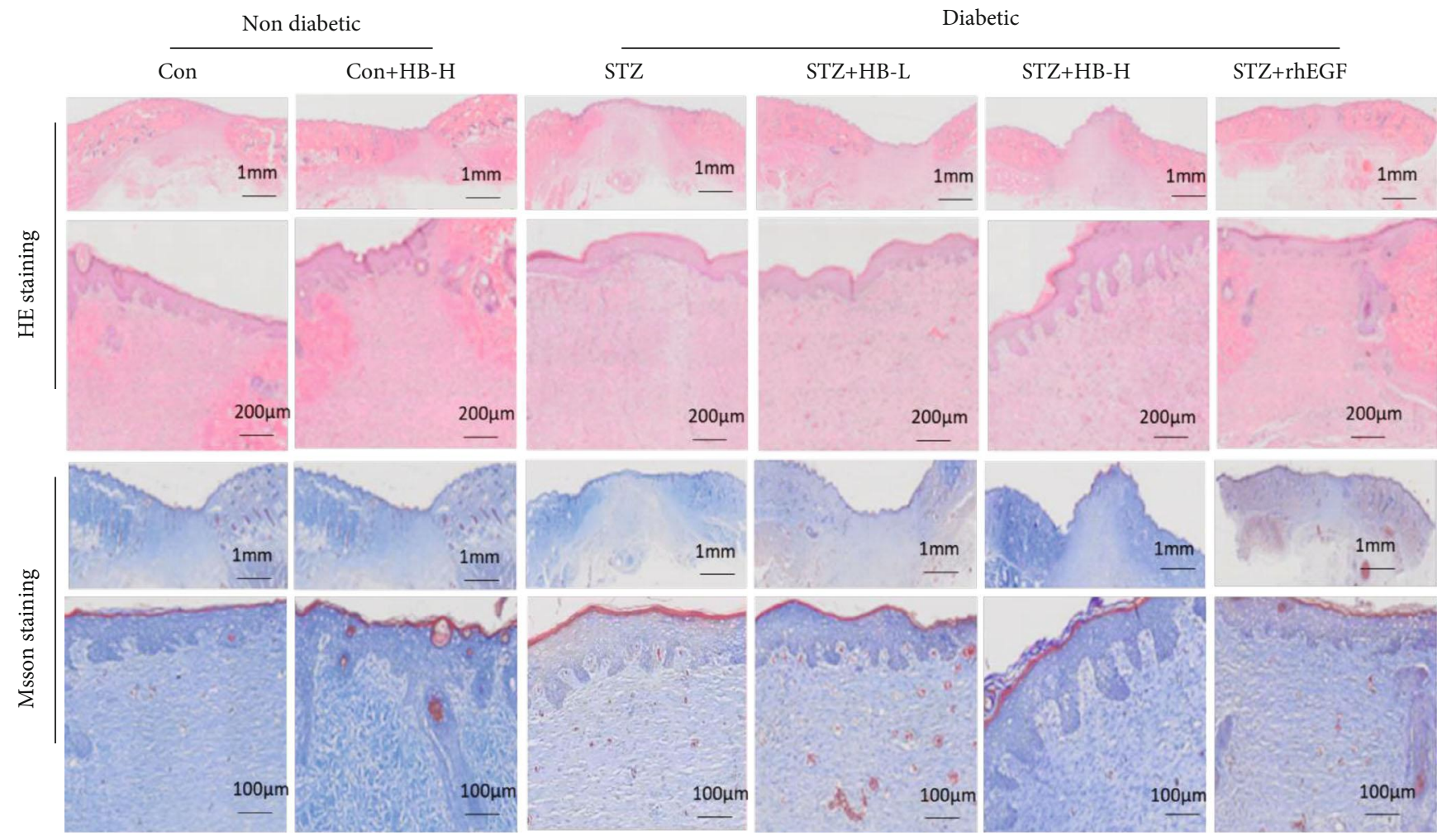

(d)

FIgURE 1: HB treatment accelerated wound closure in STZ-induced diabetic model. (a) Schematic showing diabetes induction and wound surgery generation treated with HB liniment for 13 days. (b) Representative photos of wounds healing in different time points with different treatments. (c) Wound closure analyzed by ImageJ $(n=10)$. Data were expressed as mean \pm SD, and significance was expressed as ${ }^{\#} P<0.05$ vs Con and ${ }^{*} P<0.05$ vs STZ. (d) Hematoxylin and eosin staining and Masson staining of the wound healing tissues at day 13 after wound surgery.

\subsection{The Mechanism of HB-Mediated Wound Healing Was} Systematically Investigated by RNA-Seq Technology. To reveal the underlying mechanism, RNA-seq technology was applied to systematically investigate the gene expression after various treatments. In contrast to the control, there were 4088 upregulated and 4381 downregulated genes in the STZ group. HB liniment significantly upregulated 3250 genes and downregulated 3301 genes when compared to the STZ group (Figure 2(a)). And the gene pattern of the STZ+HB group was more similar to the control group rather than the STZ group (Figure 2(b)). Additionally, the DEs between STZ and control were enriched into overrepresented GO terms including "response to oxidative stress," "cell death response to oxidative stress," "regulation of cytoskeleton organization," "cell death," "apoptotic signaling pathway," and so on (Figure 3,). Whereas, skin growth-related GO terms such as "skin development," "regulation of extracellular matrix organization," "connective tissue development," and "epithelial cell proliferation" were observed after HB treatment, indicating HB treatment improved wound healing in STZ-induced diabetic model. Importantly, oxidative stress-related GO terms such as "reactive oxygen species metabolic process" and "response to oxidative stress" and cell migration-related GO terms such as "positive regulation of locomotion" and "epithelium migration" were also found in the STZ+HB group. These data indicated oxidative stress and extracellular matrix secretion may be important factors in HB-mediated wound healing.

3.3. HB Liniment Inhibited Oxidative Damage and Apoptosis in STZ-Induced Diabetic Rats. To verify the antioxidant capacity of $\mathrm{HB}$ in diabetic rats, the amount of MDA and 8-OHdG was measured accordingly. As indicated by Figure 4(a), increased oxidative products such as MDA and 8-OHdG were observed in the STZ group. However, the increased MDA and 8-OHdG in the STZ group was reduced by $\mathrm{HB}$ and rhEGF treatment. The immunofluorescence staining also confirmed the lower staining of 8 -OHdG in the $\mathrm{STZ}+\mathrm{HB}$ group than that of the STZ group. However, no significance was found between the control group and the Con $+\mathrm{HB}$ group (Figure 4(b)). Moreover, cell apoptosis was also repressed by $\mathrm{HB}$ liniment and rhEGF as indicated by the less staining of cleaved caspase 3 than that of the STZ group (Figure 4(c)). Taken together, oxidative damage and cell apoptosis were significantly inhibited by $\mathrm{HB}$ liniment and rhEGF.

3.4. HB Liniment Activated Nrf2 and Its Downstream Genes in STZ-Induced Diabetic Rats. Since nuclear factor erythroid-derived 2-like 2 (Nrf2) plays as central regulator in the maintenance of redox homeostasis by recognizing antioxidant response element and activates antioxidant genes, the Nrf2 level and its downstream NQO1 were 

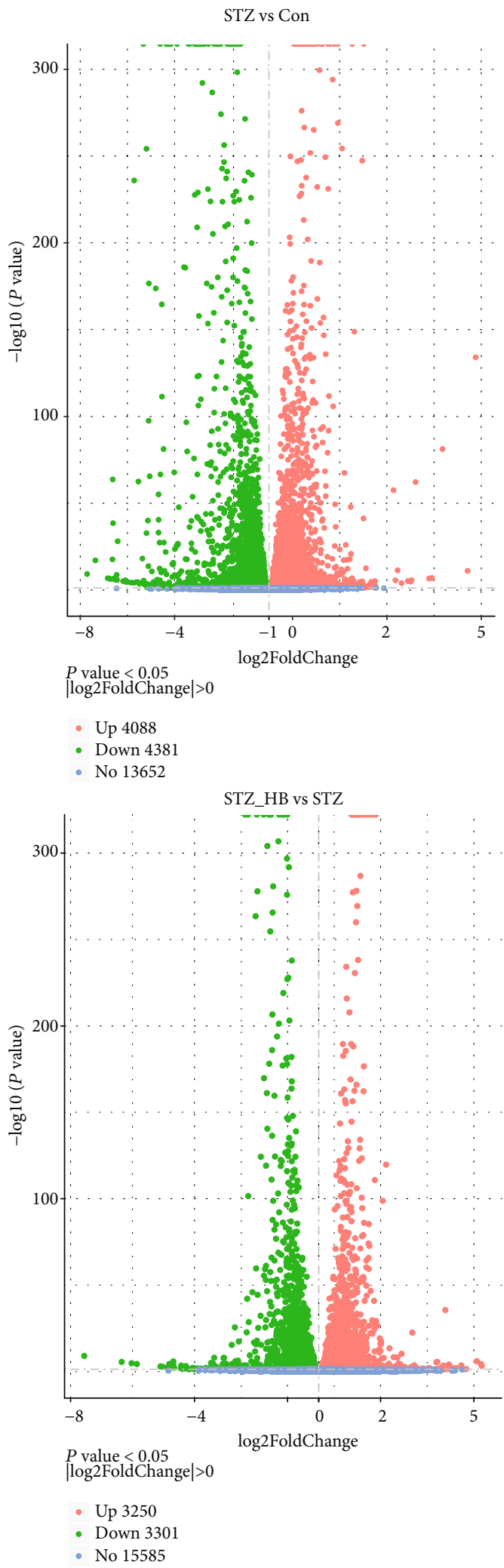

(a)

Figure 2: Continued. 


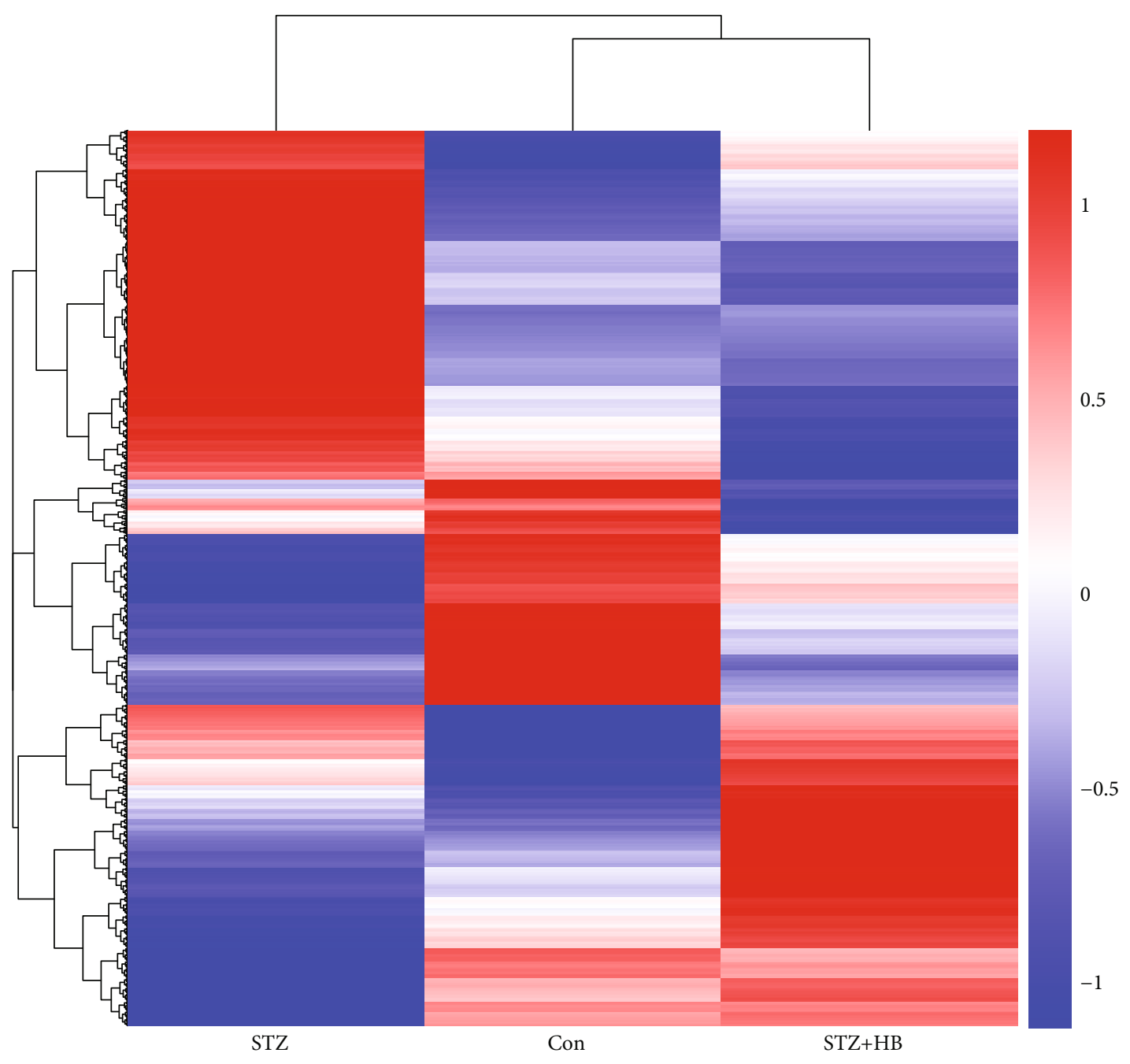

(b)

FIgURE 2: Gene expression profiling of HB-mediated wound healing in STZ-induced diabetes. (a) Differentially expressed genes (DEs) in the STZ group compared with the Con group (nondiabetic rats) and HB-treated group compared with the STZ group; upregulated DEs were shown as red dots, and downregulated DEs shown green with no significant genes as blue dots; (b) hierarchical clustering of differentially expressed genes presented as a heat map of different groups.

determined accordingly. As indicated in Figures 5(a) and 5(b), STZ group had an obvious reduction in Nrf2 expression, which was significantly reversed by $\mathrm{HB}$ liniment and rhEGF treatment. Importantly, the nuclear staining of Nrf2 in STZ-induced diabetic rats was obviously weak whereas it was enhanced by HB liniment. Additionally, the decreased NQO1 in the STZ group was also enhanced by $\mathrm{HB}$ liniment and rhEGF (Figures 5(b) and 5(c)).

To further verify the activity of Nrf2, its downstream DE genes which were involved in resisting oxidative stress were listed in Figure 6. A large number of Nrf2 downstream antioxidant genes such as Gclc, Slc7a11, Glrx5, Ggt1, Glrx3, Gsta4, Gpx1, Prdx1, Srxn1, Txnrd1, Tkt, G6pd, Taldo1, Idh1, Sod1, Sod3, and NQO1 were significantly repressed in the STZ group. Importantly, GSH-based antioxidant genes including Gclc, Gclm, Slc7a11, Glrx5, Ggt1, Glrx3, Gsta4, and Gpx1; Trx-based antioxidant genes including Prdx1, Srxn1, and Txnrd1; NADPH generation-related antioxidant genes including Me1, Tkt, G6pd, Taldo1, and Idh1, as well as other antioxidant genes including Sod1, Sod3, and NQO1 were all activated by HB liniment. Moreover, a close relationship of Nrf2 and its downstream genes was observed (Figure 6(c)). Thus, HB liniment treatment enhanced $\mathrm{Nrf} 2$ and its downstream antioxidant genes to attenuated oxidative damage.

3.5. Identification of Critical Components in HB That Affected Nfe2l 2 and Its Downstream Genes by Network Pharmacology Analysis. To identify the components which affected Nfe2l2 and its downstream differentially expressed genes (DEs), drug targets of $\mathrm{HB}$ liniment were associated with $\mathrm{Nfe} 2 \mathrm{l} 2$ and its downstream DEs and a network of componentsdrug targets-DE genes was constructed. As indicated by Figure 7, 54 components in $\mathrm{HB}$ liniment were identified through 169 drug targets by associating with Nfe2l2 and its downstream DEs. Specifically, 21 components from Cortex 

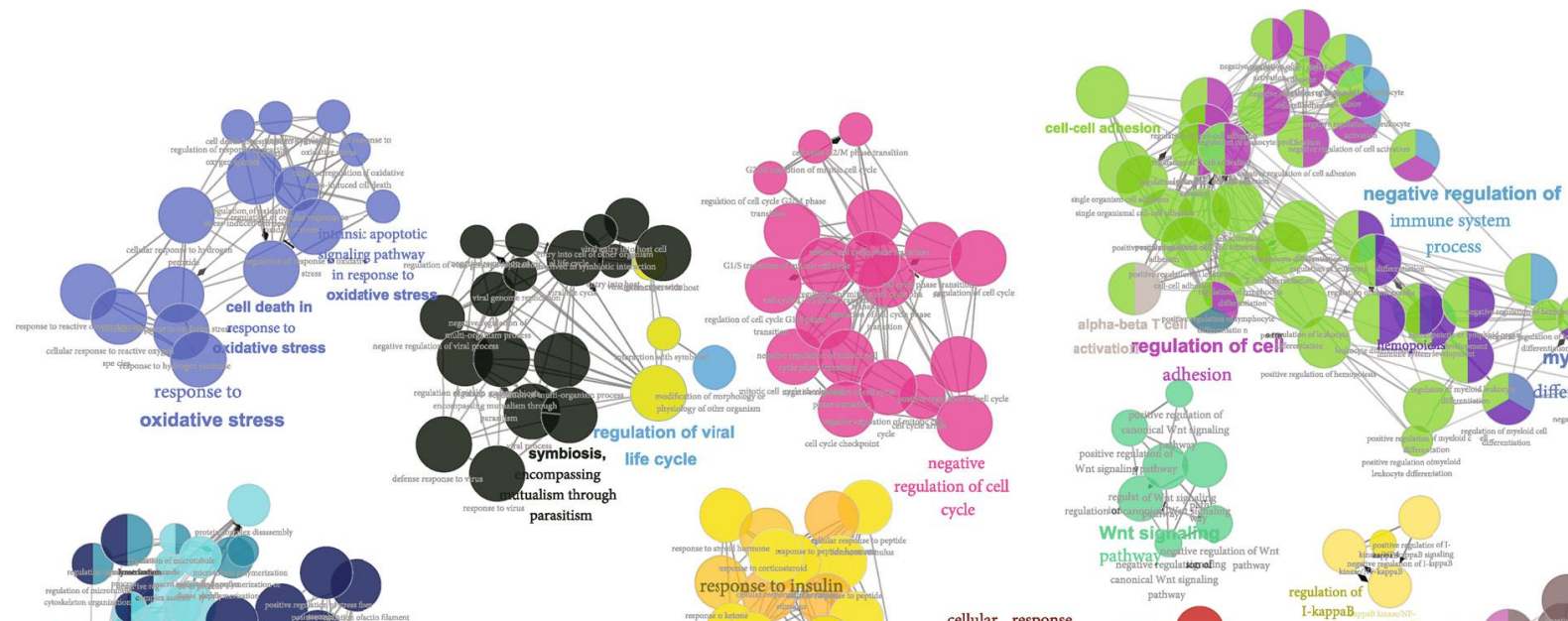

cellular respo
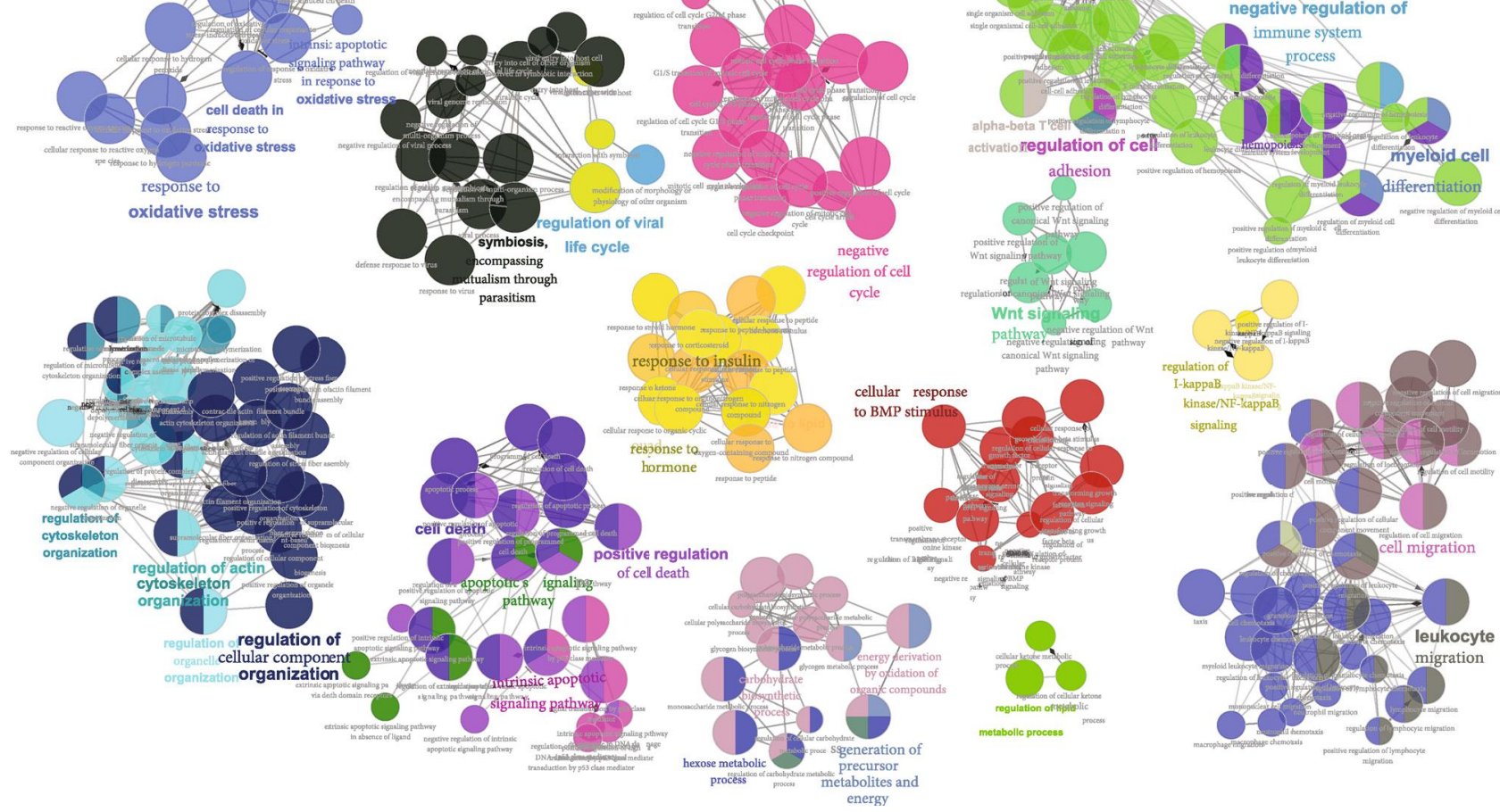

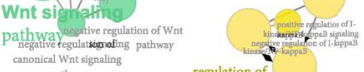

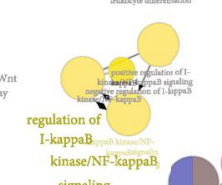

(a)
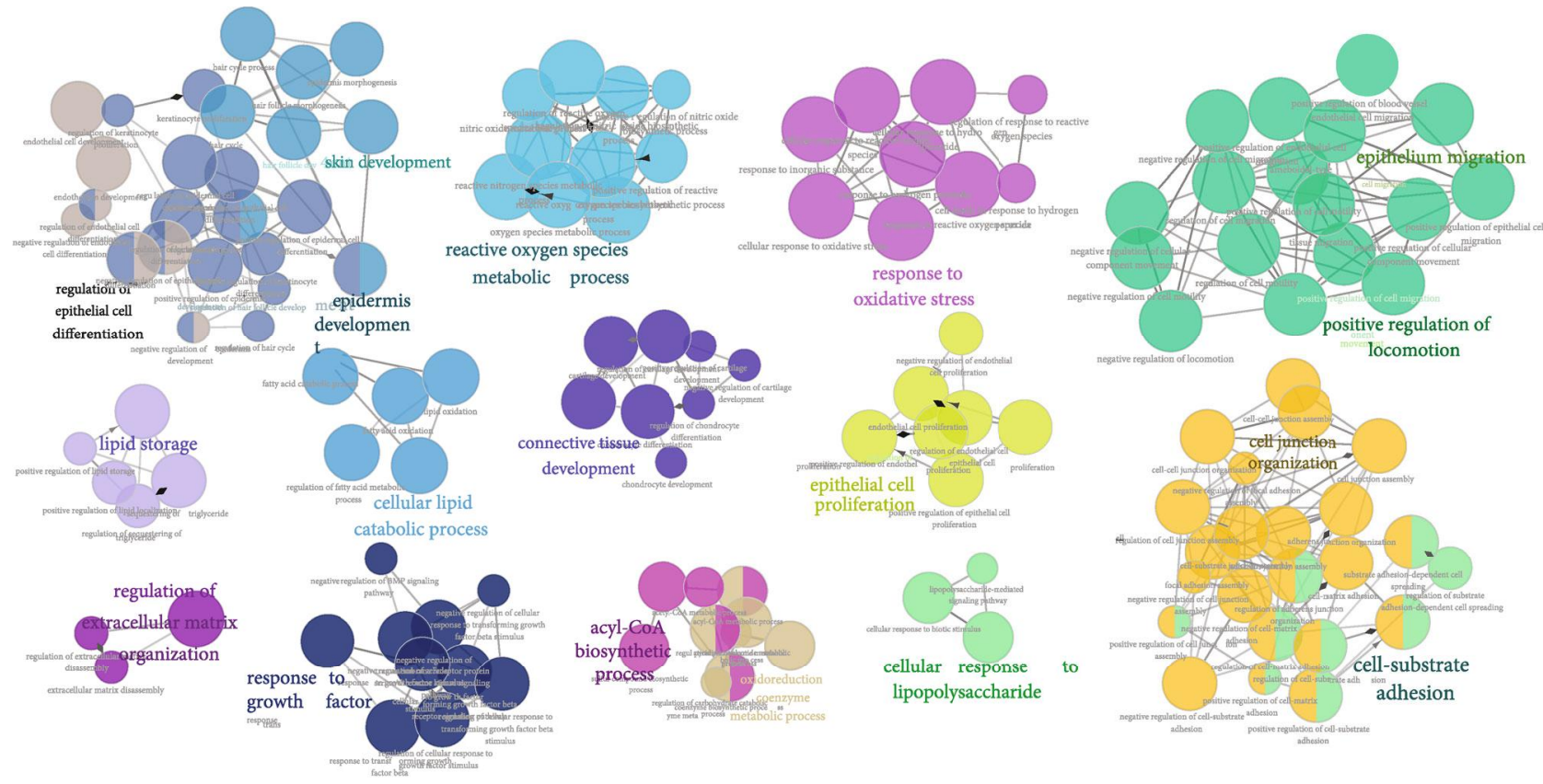

(b)

Figure 3: Enrichments of differentially expressed genes in HB-mediated wound healing by using ClueGo within Cytoscape software. (a) Enriched biological processes of DEs between the STZ group and the Con group; (b) enriched biological processes of DEs between the $\mathrm{STZ}+\mathrm{HB}$ group and the STZ group. 

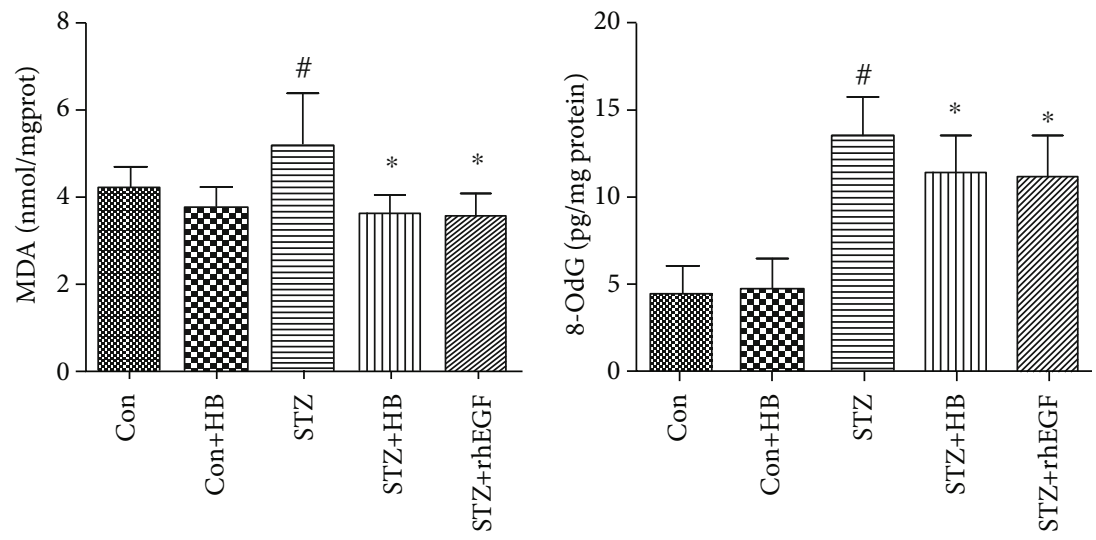

(a)
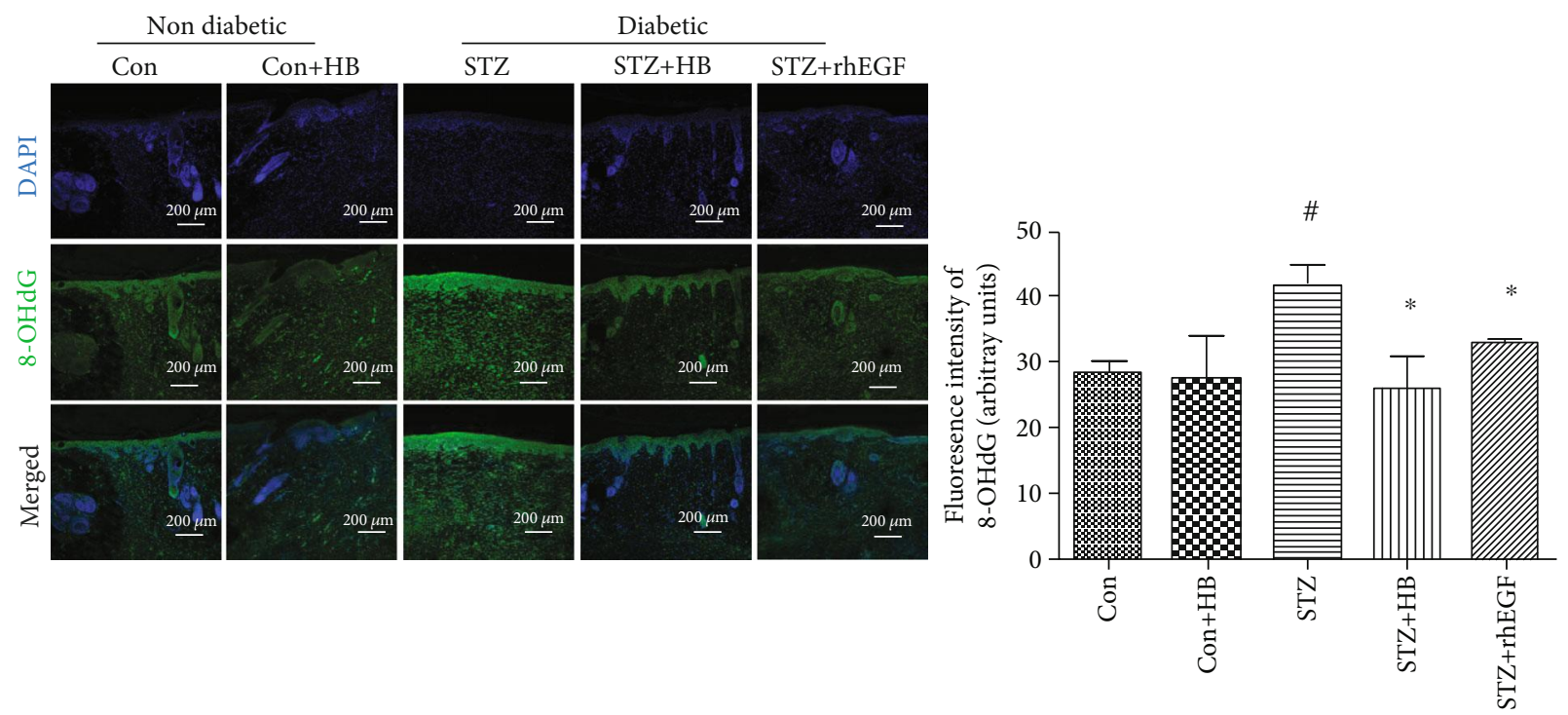

(b)
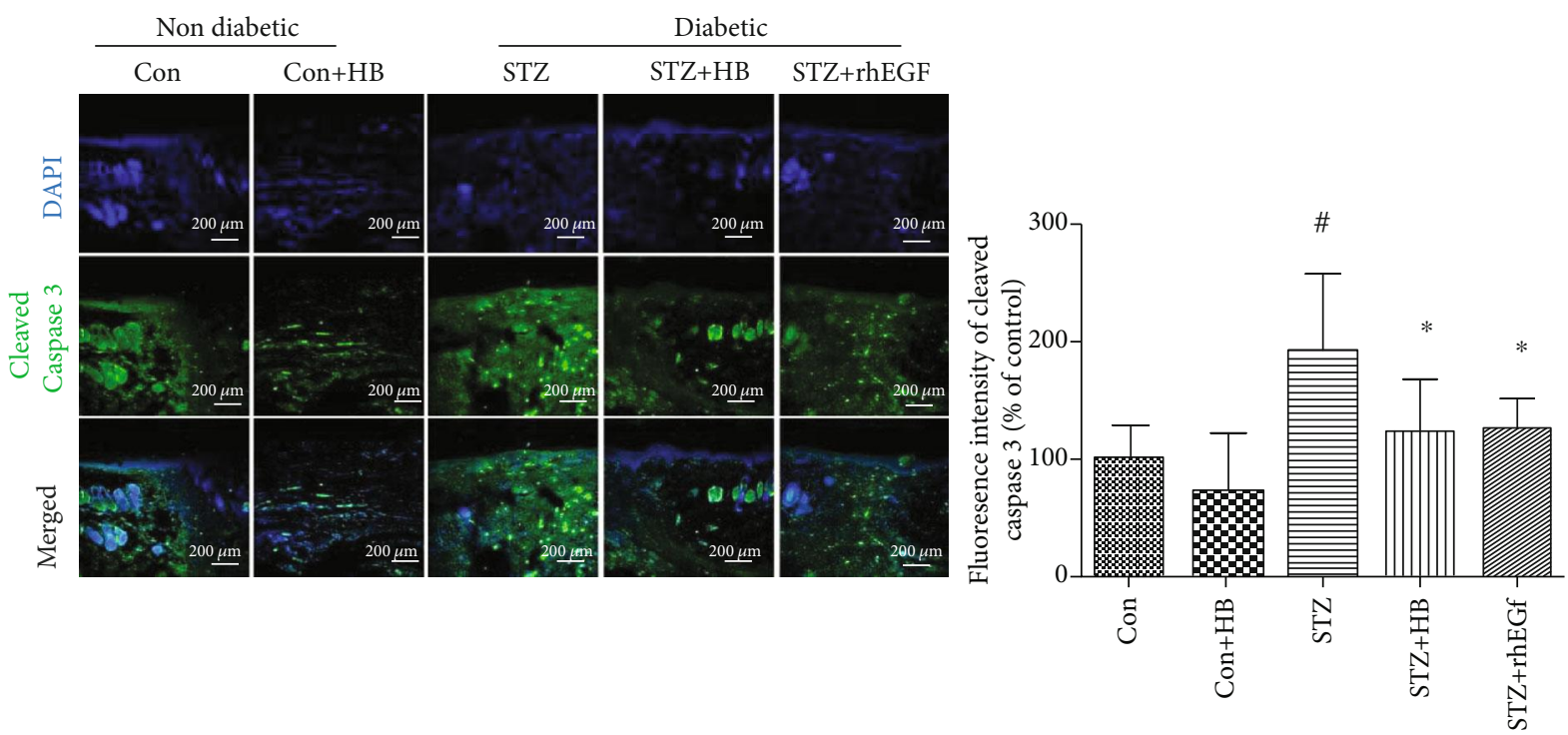

(c)

FIgURE 4: HB treatment inhibited oxidative damage and reduced cell apoptosis in diabetic wound model. (a) The level of MDA and 8-OHdG $(n=6)$; (b) immunofluorescence staining of $8-\mathrm{OHdG}$ (green) and its quantitative results $(n=3-5$ animals per group); scale bar: $200 \mu \mathrm{m}$, nucleus (blue); (c) immunofluorescence staining of cleaved caspase 3 (green) and its quantitative results $(n=3-5$ animals per group); scale bar: $200 \mu \mathrm{m}$, nucleus (blue); The data were expressed as mean \pm SD and significance was expressed as ${ }^{*} P<0.05$ vs Con and ${ }^{*} P<0.05$ vs STZ. 


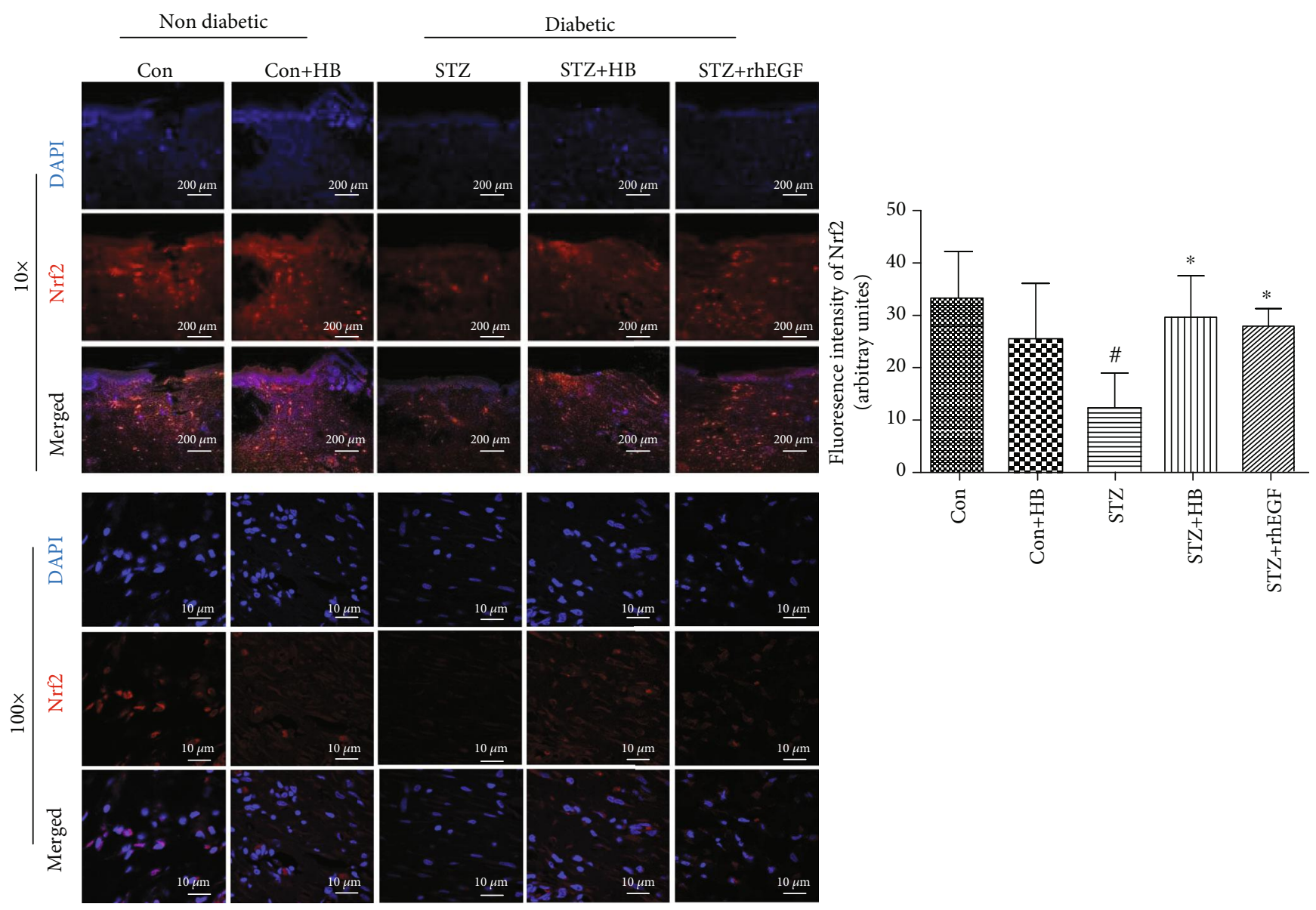

(a)

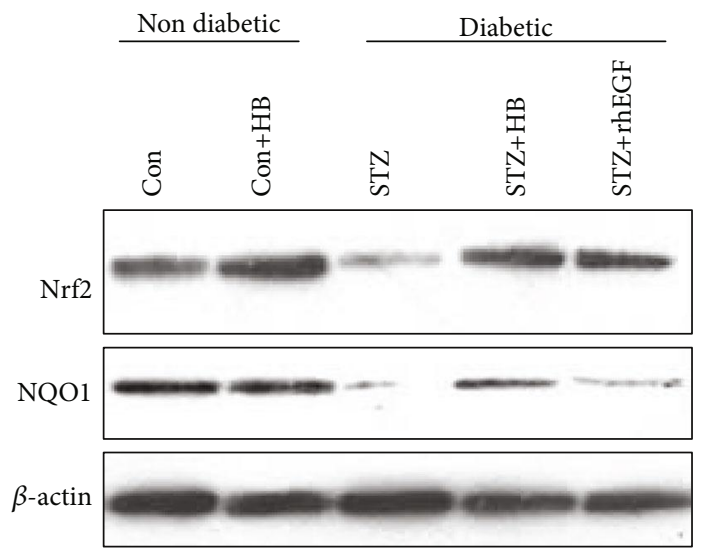

(b)

FIgure 5: Continued. 


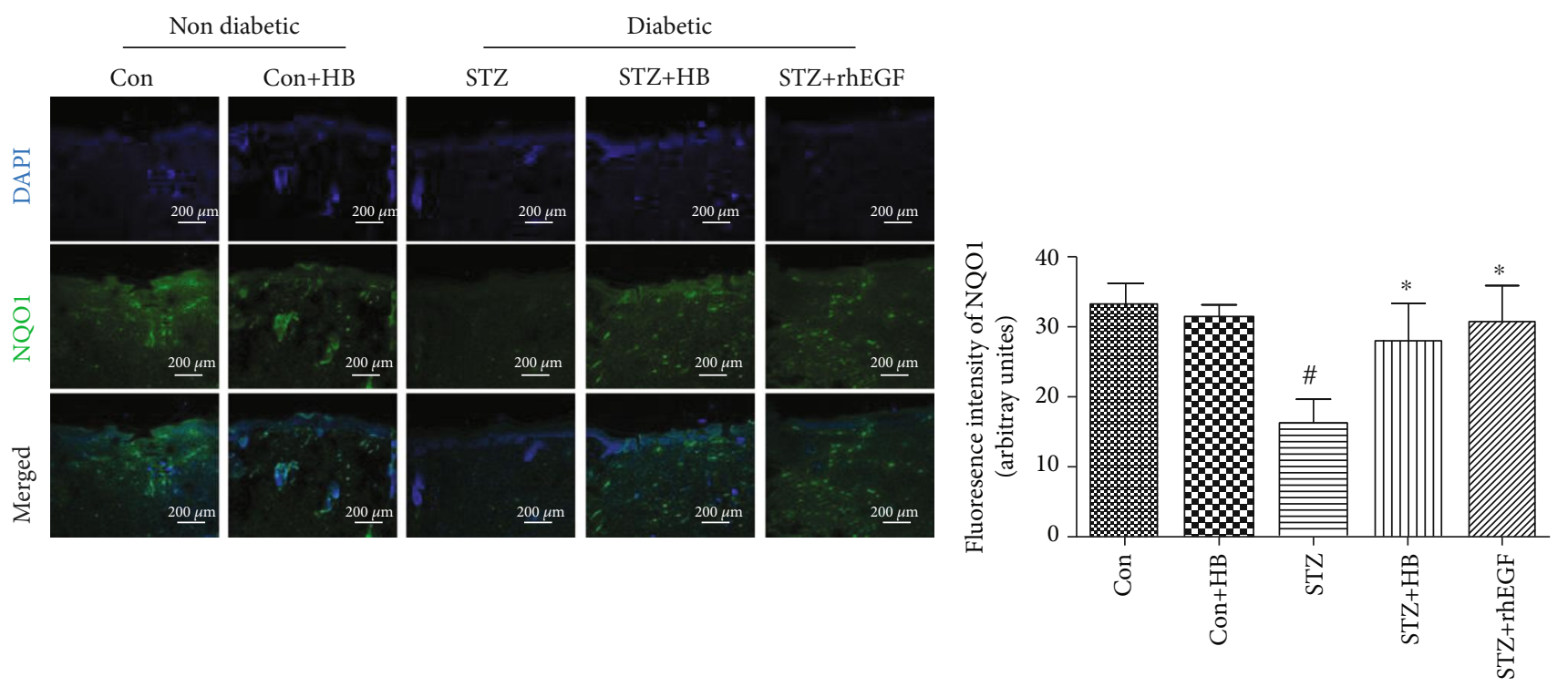

(c)

FIGURE 5: HB treatment-activated Nrf2 and its downstream NQO1 in STZ-induced wound model. (a) immunofluorescence staining of Nrf2 (red) and its quantitative results ( $n=3-5$ animals per group); nucleus (blue); (b) the representative western blotting results of Nrf2 and NQO1; (c) immunofluorescence staining of cleaved caspase 3 (green) and its quantitative results $(n=3-5$ animals per group); scale bar: $200 \mu \mathrm{m}$, nucleus (blue); the data were expressed as mean $\pm \mathrm{SD}$, and significance was expressed as ${ }^{\#} P<0.05$ vs Con and ${ }^{*} P<0.05$ vs $\mathrm{STZ}$.

Phellodendri including Cinnamic acid, caffeic acid, Jatrorrhizine, 3,4-dihydroxybenzoic acid, sanleng acid, and 16 other components while 18 components from Forsythia suspensa including suspensine A, hydroxytyrosol, vanillic acid, Succinic acid, and other 14 components were screened out. Additionally, there were 9 components from Taraxacum mongolicum Hand.-Mazz, 5 from Lonicera japonica Thunb, and 7 from Scolopendridae were identified to have an effect on the differentially expressed oxidative genes. Among these identified components, there were several components originated from multiple drugs. For example, Quercetin generated from both Forsythia suspense, Lonicera japonica Thunb, and Taraxacusm mongolicum Hand.-Mazz.

3.6. HB Liniment Modulated the Expression of MMP9 and TGF- $\beta 1$ and Improved Collagen Growth. To further reveal the mechanism of HB, TGF- $\beta 1$, MMP9 level, and the collagen growth were evaluated accordingly. As indicated by Figure 8, the decreased TGF- $\beta 1$ and elevated MMP9 in the STZ group were significantly reversed by HB liniment and rhEGF. The neo-collagen growth at the front of the wound healing was increased by both HB liniment and rhEGF in contrast to the STZ group. These results showed HB liniment and rhEGF enhanced TGF- $\beta 1$, reduced MMP9, and improved collagen growth.

3.7. HB Liniment and Quercetin Promoted Cell Proliferation and Decreases Oxidative Damage in HG-Induced Model. To further confirm the mechanism, the effect of $\mathrm{HB}$ and quercetin (one of its components), which was reported to have an effect on diabetic wounds, was tested using $\mathrm{HaCaT}$ cells. As indicated by Figure $9, \mathrm{HB}$ and quercetin did not inhibit cell viability but enhanced cell proliferation in low-glucose medium. HG stimulation caused a remarkably decrease in cell viability, whereas this was ameliorated by $\mathrm{HB}$, quercetin, and povidone iodine. To confirm the effect of $\mathrm{HB}$ on oxidative stress, the levels of ROS and GSH were measured accordingly. HG stimulation led to a significant decrease in GSH level, which was dramatically increased by $\mathrm{HB}$ and quercetin. Additionally, the increased ROS level induced by HG was also significantly reduced by $\mathrm{HB}$ and quercetin but not by povidone iodine. The positive rate of Ki67 (a cell proliferation marker) was decreased by HG stimulation, which was significantly increased by $\mathrm{HB}$, but not by quercetin or povidone iodine (Figure 9(c)). These data indicated that HB had a good effect in attenuating oxidative damage and promoting cell proliferation.

\section{Discussion}

Diabetic wounds cause high mortality and disability in a large number of diabetic patients, whereas there is a limited effective therapy. In this study, the effect of Huangbai (HB) liniment on STZ-induced diabetic wounds was systematically investigated, and the mechanism was revealed. HB liniment significantly accelerated the wound closure and enhanced the generation of extracellular matrix in STZ-induced diabetic rats. By using RNA-seq technology analysis, oxidative stress was identified as a vital process in HB-mediated wound healing. Importantly, the activation of Nrf2 and its downstream genes involved in glutathione system, thioredoxin system, and NADPH generation as well as other antioxidant genes were observed after $\mathrm{HB}$ treatment. The activated Nrf2 and its downstream genes developed a stable antioxidant network, contributing to the reduction of oxidative damage and apoptosis (Figure 10). Moreover, HB liniment enhanced 


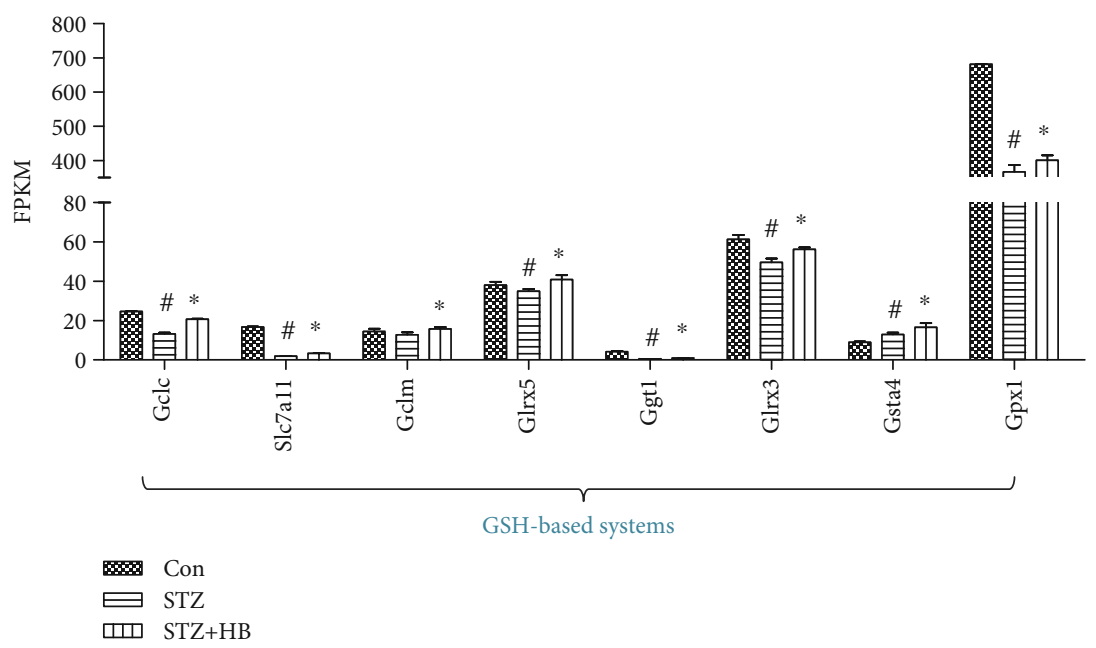

(a)

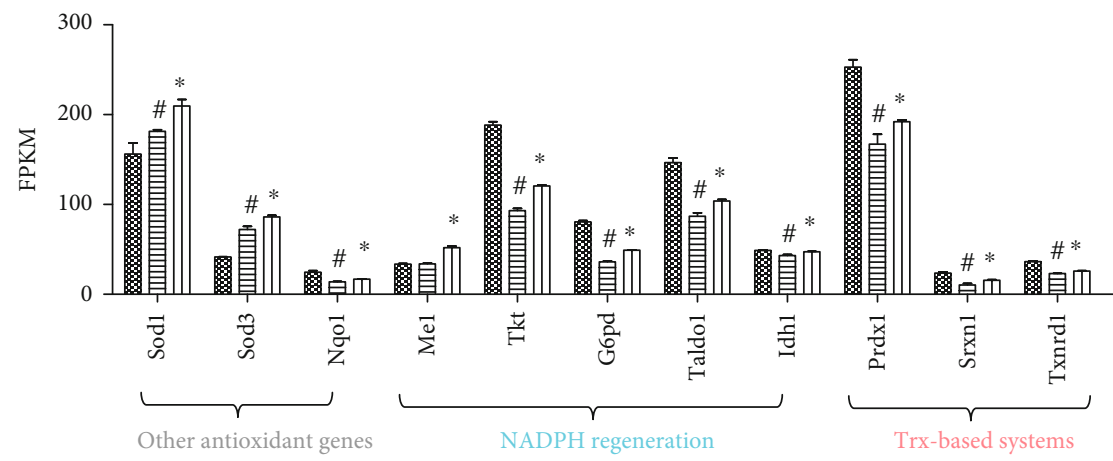

\%... Con

$\rightleftharpoons \mathrm{STZ}$

سा STZ+HB

(b)

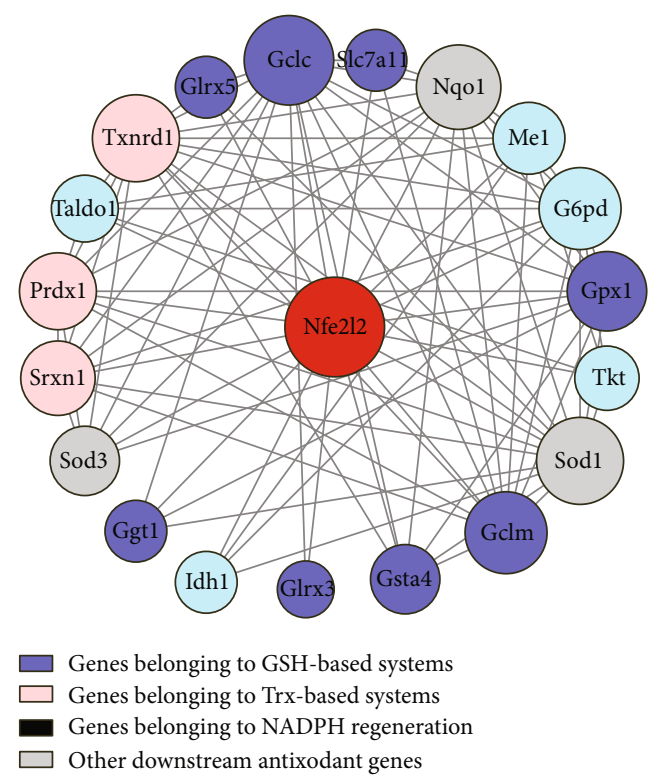

(c)

FIGURE 6: HB treatment activated the downstream genes of Nrf2 in diabetic wound model. (a) The downstream genes of Nrf2 involved in GSH-based antioxidant systems $(n=3)$; (b) The downstream genes of Nrf2 involved in Trx-based antioxidant systems, GAPDH generation and other antioxidant processes $(n=3)$; (c) The network of Nfe2l2 and its downstream DEs constructed by Cytoscape software. The data were expressed as mean $\pm \mathrm{SD}$, and significance was expressed as ${ }^{\#} P<0.05$ vs Con and ${ }^{*} P<0.05$ vs STZ. 


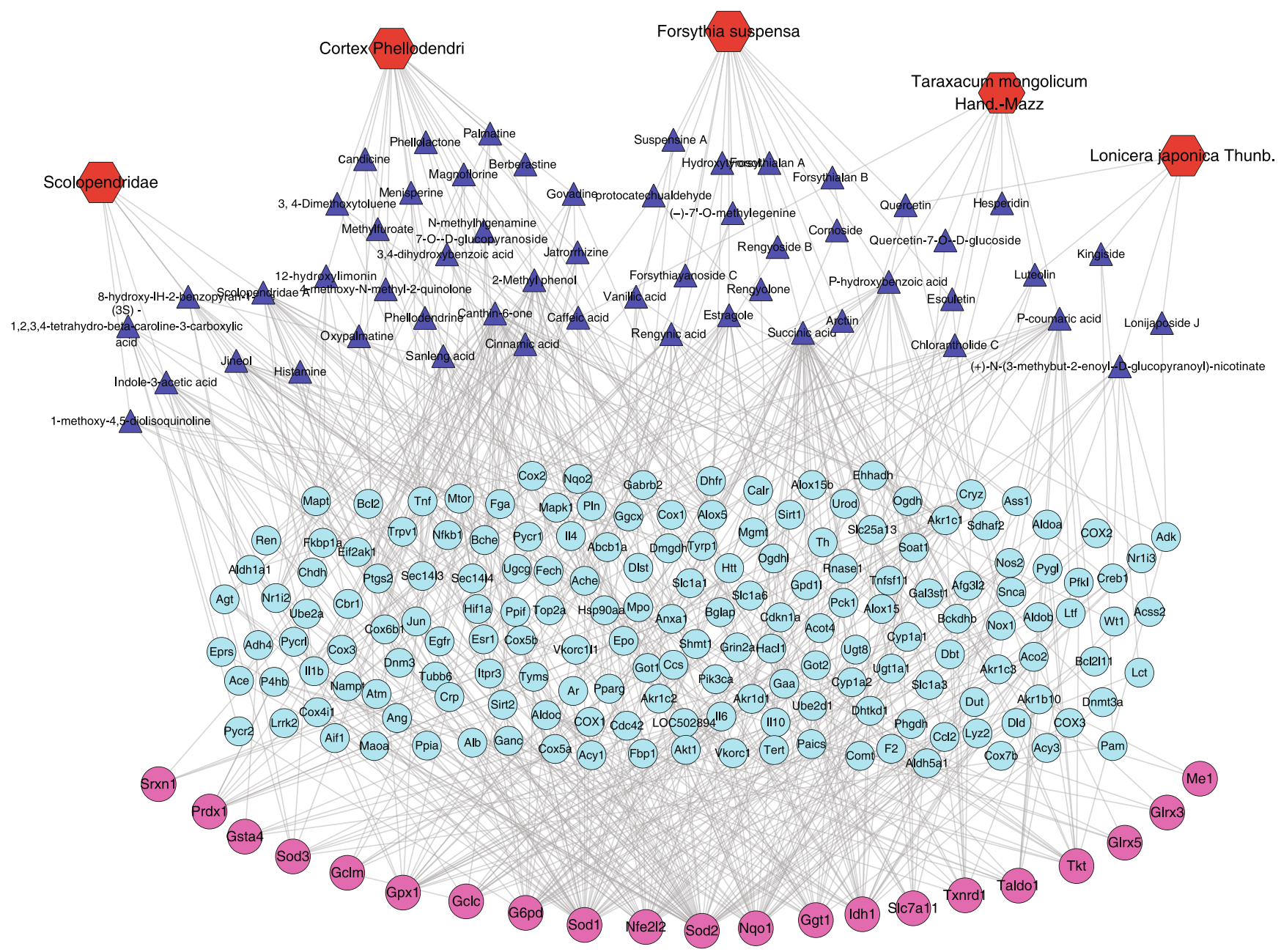

FIgURE 7: The components of HB liniment which affected Nfe2l2 and its downstream differentially expressed genes (DEs) were identified through Network pharmacology analysis. Specifically, 54 components in HB liniment were identified with 21 components from Cortex Phellodendri, 18 components from Forsythia suspensa, 9 components from Taraxacum mongolicum Hand.-Mazz, 5 from Lonicera japonica Thunb., and 7 from Scolopendridae. Some components generated from multiple Chinese medicine. Red hexagons indicated the Chinese medicine, blue triangles indicated the components of $\mathrm{HB}$, blue dots indicated drug targets of components from HB liniment, and red dots indicated Nfe2l2 and its downstream DEs.

TGF- $\beta 1$, reduced MMP9 level, and promoted collagen growth, thereby accelerating wound healing in diabetic conditions. The in vitro experiment demonstrated the good effect of $\mathrm{HB}$ in inhibiting oxidative damage and enhancing cell proliferation. By associating drug targets of $\mathrm{HB}$ liniment with Nrf2 and its downstream differentially expressed genes, 54 components were screened out, and the majority of the components was from Cortex Phellodendri and Forsythia suspensa.

Growing evidence has accumulated that redox homeostasis serves a significant role in modulating diabetic wound healing [23]. Overproduced ROS in diabetes damages macromolecules such as lipids, proteins, and DNA double strands, thus results in impaired wound healing and apoptosis. In our study, oxidative stress was identified as a vital process in HBmediated wound healing (Figure 3 ), which was subsequently confirmed by decreased high DNA damage (8-OHdG), peroxide products (MDA), and reduced apoptosis in STZ rats. As a central regulator of cellular redox status, Nrf2 is respon- sible for modulating transcription of cytoprotective and antioxidant genes, which affects the ability of wounds to heal in diabetics [24]. Activation of Nrf2 by its activator or inhibiting its repressor Keap1 alleviated ROS generation, decreased oxidative damage, and accelerated wound closure in diabetes $[25,26]$. On the contrary, $\mathrm{Nrf} 2^{-/-}$mice was characterized by delayed wound healing [25], indicating the vital role of Nrf2 in facilitating wound closure in diabetes. As consistently demonstrated in our study, hindered Nrf2 activity in diabetes failed to modulate redox homeostasis and resulted in impaired wound closure, whereas the Nrf2 signaling restored by $\mathrm{HB}$ treatment inhibited oxidative damage and promoted wound healing. In addition, our results demonstrated the dysfunction of its target genes involved in GSH-based, Trxbased antioxidant systems, as well as NADPH-generating process and other antioxidant genes such as NQO1, Sod1, and Sod3 were also rescued by $\mathrm{HB}$ treatment in STZinduced diabetic wound (Figure 6). Nrf2 activation enhanced expression of Gclc and Gclm which determined the GSH 


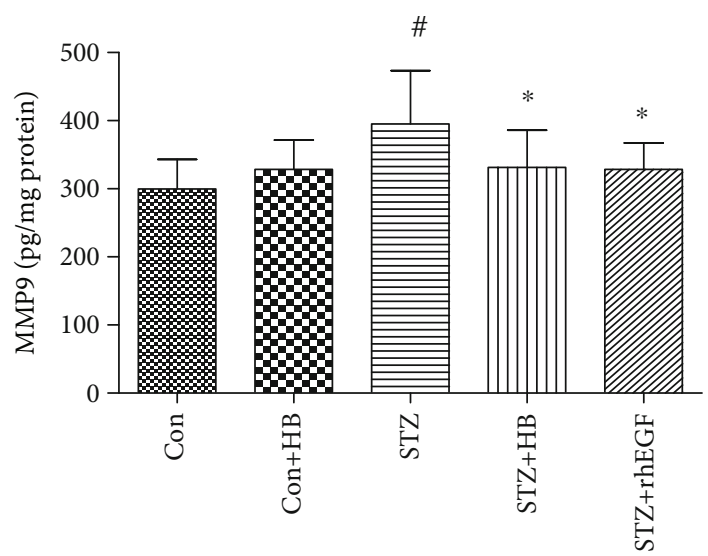

(a)

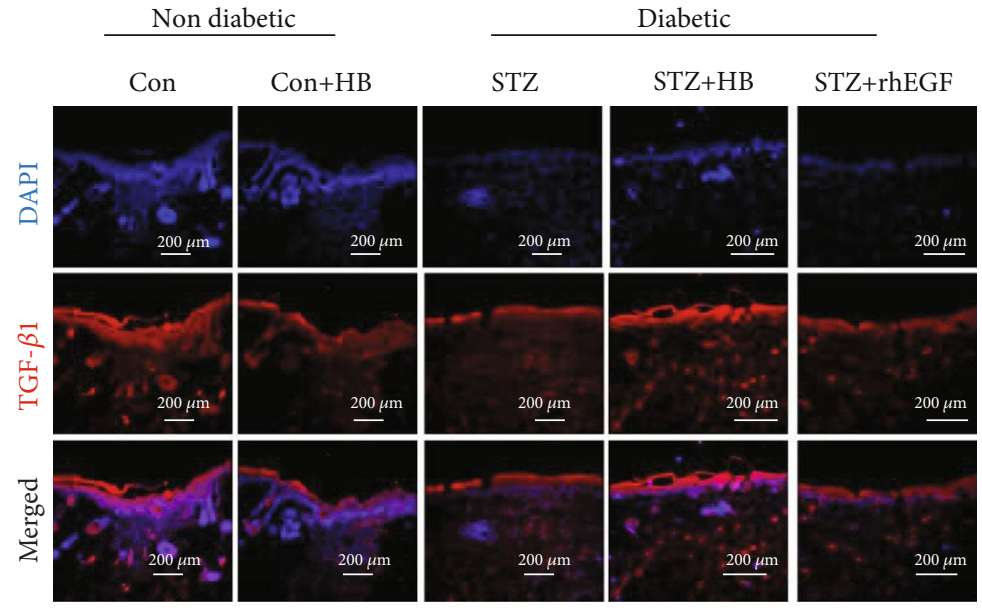

(b)

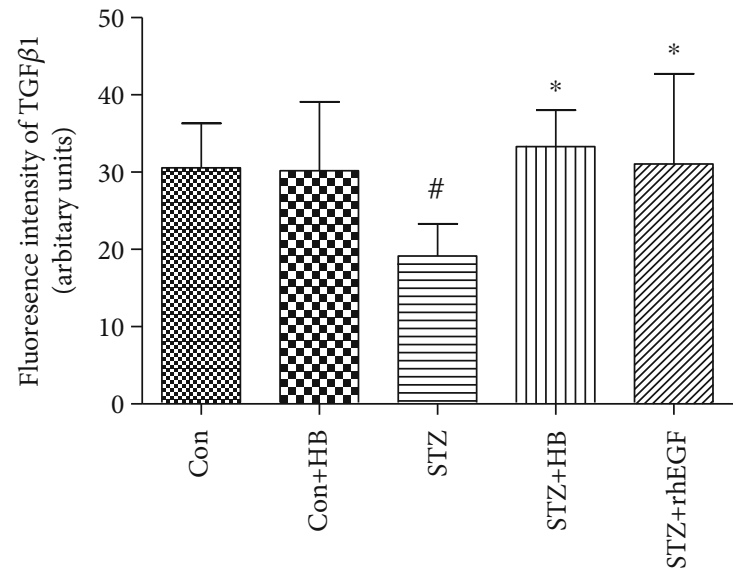

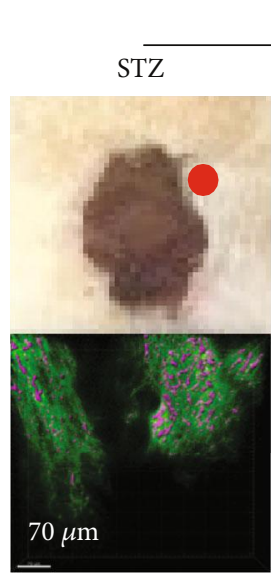

Day 6
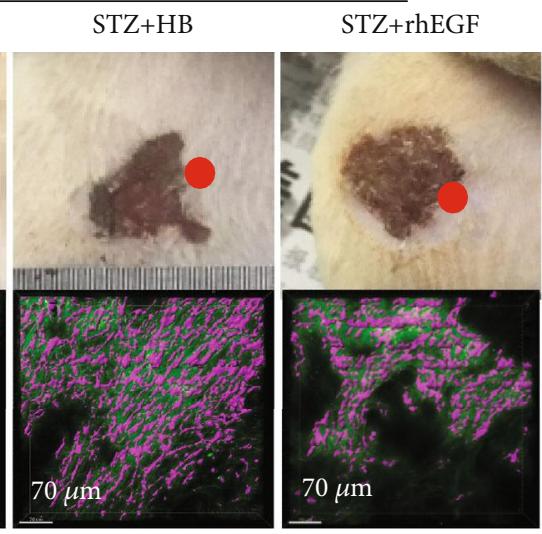

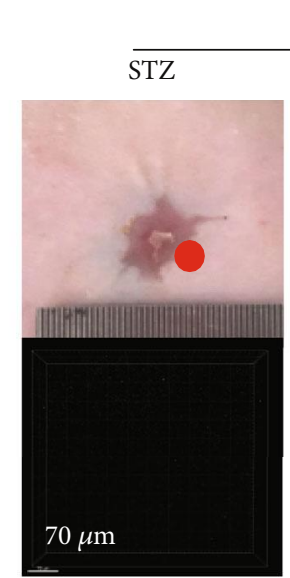

Day 13
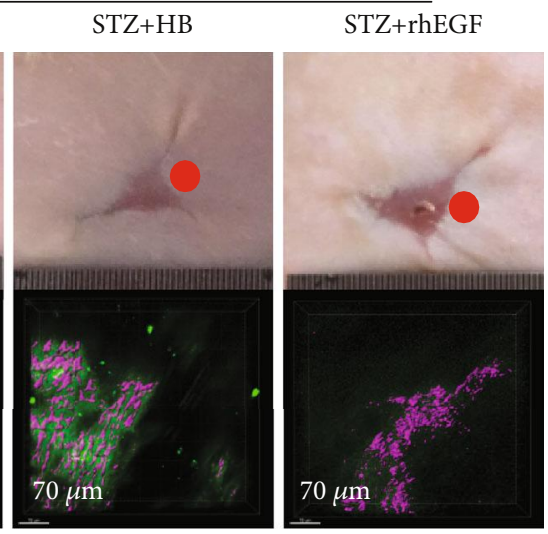

(c)

FiguRE 8: HB treatment enhanced TGF- $\beta 1$ expression, decreased MMP9 production, and facilitated collagen growth. (a) immunofluorescence staining of TGF- $\beta 1$ (red) and its quantitative results ( $n=3-5$ animals per group); scale bar: $200 \mu \mathrm{m}$, nucleus (blue); (b) MMP9 level $(n=6)$; (c) New collagen growth observed by using two-photon microscope at days 6 and 13 after wound surgery. The green indicated the background, and the purple indicated the new collagen tissue at the front of the wound healing. The data were expressed as mean $\pm \mathrm{SD}$, and significance was expressed as ${ }^{\#} P<0.05$ vs Con and ${ }^{*} P<0.05$ vs STZ. 

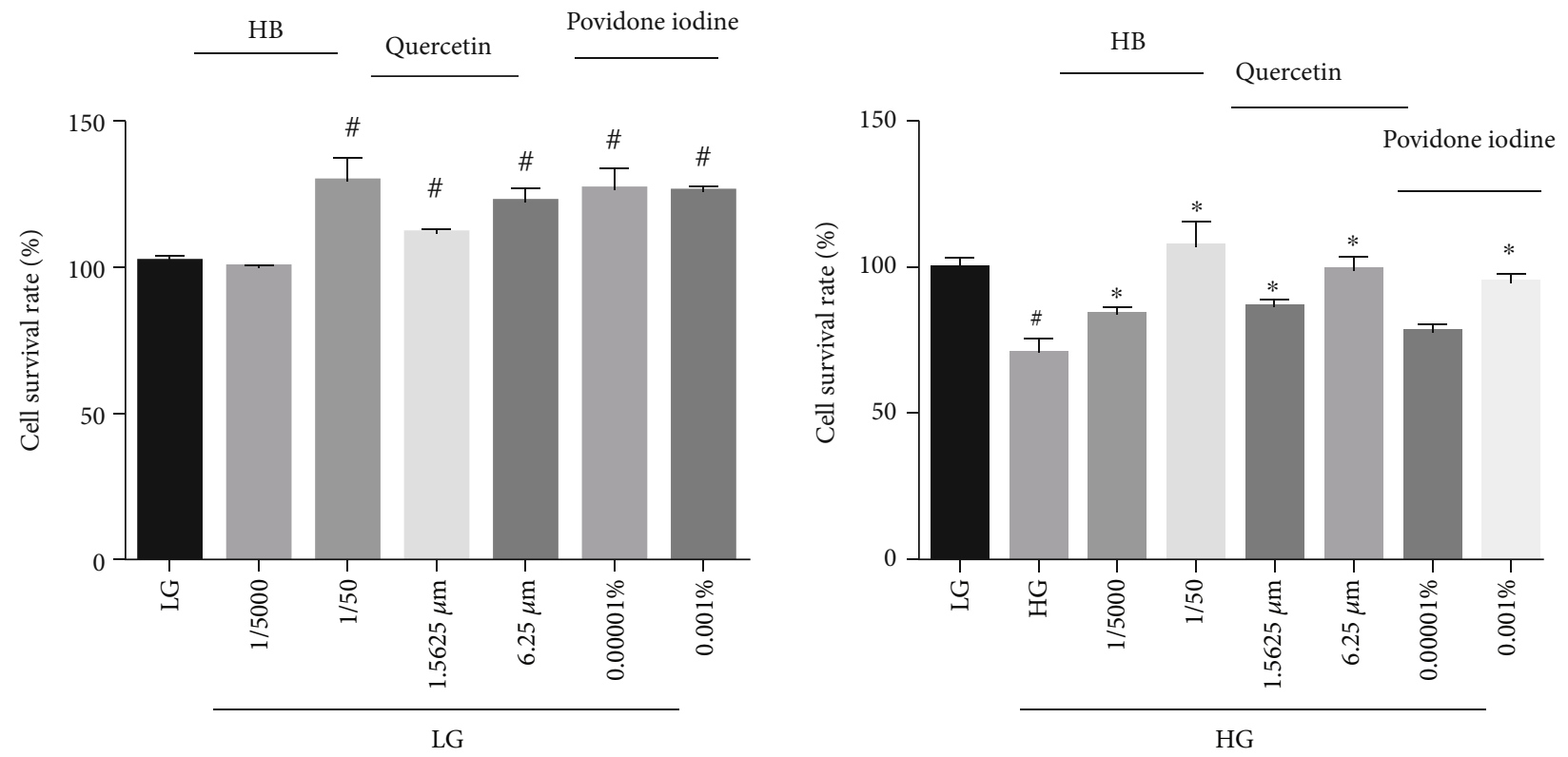

(a)
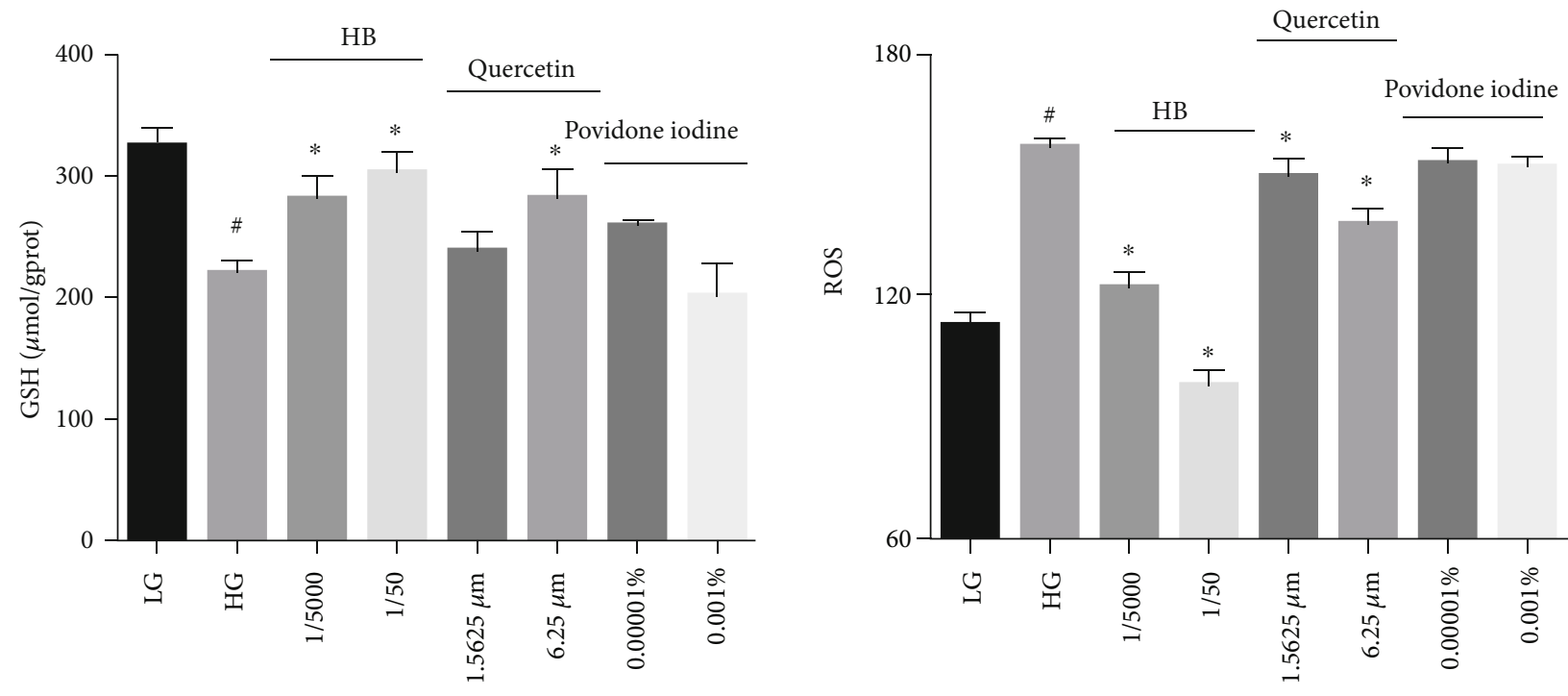

HG

HG

(b)

Figure 9: Continued. 


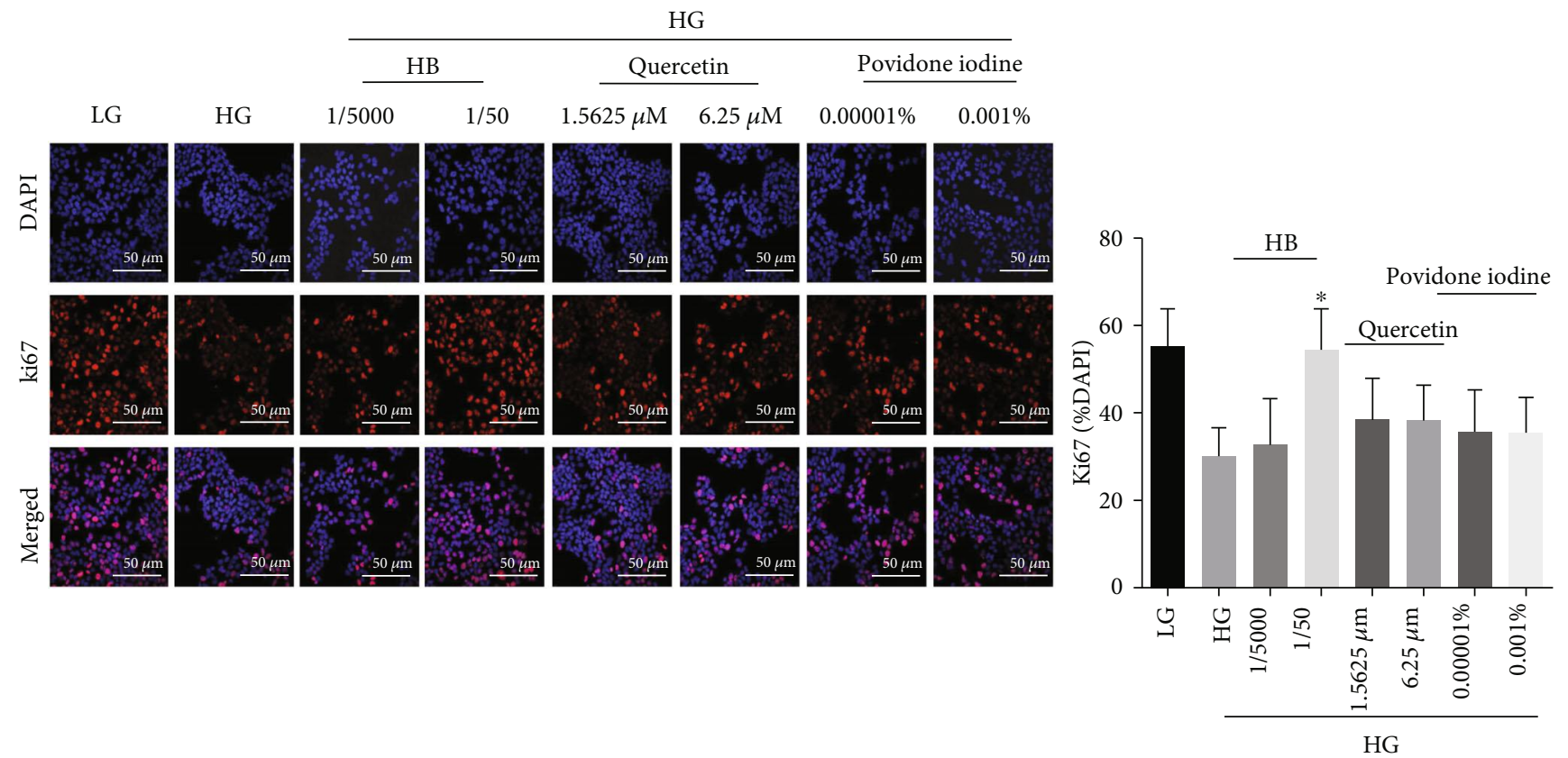

(c)

FIGURE 9: HB facilitated cell proliferation and reduced oxidative damage in HaCaT cells. (a) Cell viability in LG and HG ( $n=6$ ); (b) the levels of GSH ( $n=3)$ and ROS ( $n=6)$; (c) immunofluorescence staining of Ki67 (red) and its quantitative result ( $n=3-5$ per group), nucleus (blue), scale bar: $50 \mu \mathrm{m}$; the data were expressed as mean $\pm \mathrm{SD}$, and significance was expressed as ${ }^{*} P<0.05$ vs $L G$ and ${ }^{*} P<0.05$ vs $H G$.

biosynthesis, Gpx1 which helped to decompose $\mathrm{H}_{2} \mathrm{O}_{2}$ and produce GSSG, and Ggt1 which initiated extracellular glutathione (GSH) breakdown. The elevated levels of GSH-based antioxidant genes maintained GSH homeostasis and ensured that its antioxidant effect was realized [27]. Moreover, Nrf2 activation upregulated gene expression of Txnrd1, Srxn1, and $\operatorname{Prdx} 1$, which allowed for the reduction of oxidized thiols in proteins [28]. As a vital reducing resource, NADPH was required for the GSH and Trx regeneration to eliminate excess ROS [29]. The elevated gene expression of Me1, Tkt, Idh1, and Taldo1 by Nrf2 demonstrated the improved function of NADPH generation and maintained GSH- and Trx- based antioxidant function. Thus, the activated Nrf2, together with its downstream genes involved in GSH-based, Trx-based antioxidant systems, as well as NADPH generating process and other antioxidant genes, eliminated ROS, decreased oxidative damage and apoptosis, and accelerated wound healing in diabetes.

The compounds in HB liniment that affected Nle2l2 and its downstream genes were identified accordingly. Specifically, 54 components from HB liniment were identified to have an effect on Nle212 and its downstream antioxidant genes. Significantly, among the 21 identified components from Cortex Phellodendri, Cinnamic acid [30], caffeic acid [31], Magnoflorine [32, 33], Jatrorrhizine [34], phellodendrine, and 3,4-dihydroxybenzoic acid have proven to reduce oxidative stress. The extract of Forsythia suspensa exhibited a significant antihyperglycemic and antihyperlipidemic effect in the treatment for diabetes by modulating oxidative stress and insulin secretion [35]. The 18 compounds from Forsythia suspensa, including quercetin [36], vanillic acid [37],
Arctiin [38], hydroxytyrosol [39] and Protocatechualdehyde [40], ameliorated oxidative damage. Additionally, Taraxacum mongolicum Hand.-Mazz [41]. and its identified components such as p-Coumaric acid and Esculetin [42, 43], as well as Lonicera japonica Thunb., and its identified components such as Luteolin had a beneficial antioxidant effect by removing ROS $[44,45]$. Moreover, no studies have shown that Scolopendridae were associated with diabetes, although they have demonstrated to exhibit good antioxidant capacity [46]. In this study, the antioxidant property of HB liniment was observed in the treatment of STZ-induced diabetic wound by using the combination of Cortex Phellodendri, Forsythia suspensa, Lonicera japonica Thunb., Taraxacum mongolicum Hand.-Mazz, and Scolopendridae. In addition, quercetin, which came from three of the five compositions of HB liniment, demonstrated multiple effects in facilitating wound healing [47]. Researches showed it not only has good antibacterial action, similar to other standard drugs (e.g., povidone iodine) [48], but also decreased inflammation, inhibited oxidative damage, and enhanced secretion of VEGF and TGF- $\beta 1$, modulated integrin expressions to reduce fibrosis during wound healing $[47,49]$. In this study, the effect of $\mathrm{HB}$, quercetin, and povidone iodine on inhibiting oxidative damage was compared in HG-induced HaCaT cell model. It demonstrated that both $\mathrm{HB}$ and quercetin had a good effect in inhibiting oxidative damage rather than povidone iodine (Figure 9). Thus, four herbs and Scolopendridae in HB liniment shared some same components, and the 54 identified compounds had a synergistically inhibitive effect on oxidative stress through modulating Nrf2 and its downstream antioxidant genes. 


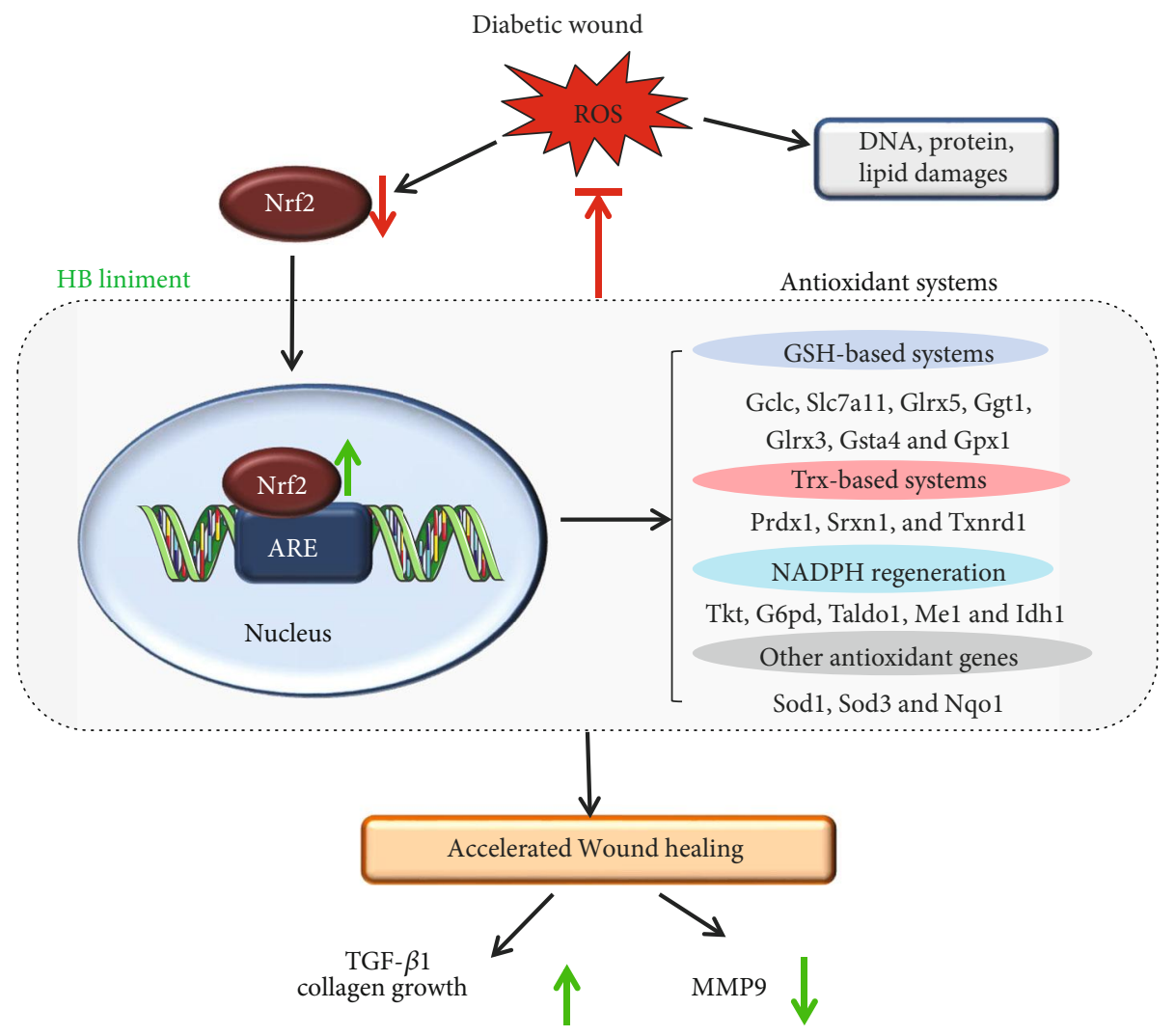

FIgURE 10: Schematic diagram illustrating of the potential mechanism of HB-mediated diabetic wound healing. Reactive oxygen species in diabetic wound caused DNA double strands breakage, lipid peroxide, protein denaturation, and impaired diabetic wound healing. Specifically, Nrf2 and its downstream antioxidant genes were significantly decreased, and wound healing was impaired. In contrast, HB liniment markedly increased Nrf2 level and its downstream genes involved in GSH- and Trx-based systems, NADPH generation, and other antioxidant systems; this led to enhanced antioxidant capacity and decreased oxidative and apoptosis damage. Additionally, these also increased TGF- $\beta 1$ and collagen growth while decreased MMP9, which accelerated wound closure in HB-mediated wound healing.

Along with the enhanced antioxidant capacity by $\mathrm{HB}$ liniment, an increase in transforming growth factor- $\beta 1$ (TGF- $\beta 1)$ and reduction in matrix metalloproteinase (MMP) contributed to the increased extracellular matrix growth and enhanced wound healing in diabetes. Elevated levels of ROS hinder tissue growth, especially for collagen production and halt wound closure [12], and the interference of ECM production is highlighted through the disruption of TGF- $\beta 1$ signaling $[10,11]$. Besides, high ROS level affects ECM remodeling through regulating MMPs expression, and ROS production is required for MMPs synthesis [13]. These were consistent with our results that serious oxidative damage in diabetic rats was associated with low ECM synthesis and collagen generation. Delayed wound healing in diabetes is usually characterized with low TGF- $\beta 1$ and high MMPs in skin tissue of patients with diabetic foot ulcer [50-52]. TGF- $\beta 1$ signaling plays a critical role as the regulator in a series of biological processes of skin regeneration and wound healing, such as angiogenesis and reepithelialization. [53]. MMP9 is responsible for eliminating damaged extracellular matrix and allows for skin tissue remodeling [54]. An overproduction of MMP9 in diabetes causes excessive ECM degradation and results in delayed wound healing [54], but inhibition or knockout of MMP9 promotes diabetic wound healing [55]. This indicates a critical role of MMP9 in treat- ing diabetic ulcers. Consistent with these research findings, decreased TGF- $\beta 1$ and elevated MMP9 were observed in our diabetic wound model, and these were significantly alleviated by $\mathrm{HB}$ treatment as indicated by significantly higher TGF- $\beta 1$ and reduced MMP9 level. Recent research indicates that Nrf2 activation could promote TGF- $\beta 1$ generation and inhibit the overproduction of MMP9 in diabetic wound [25] Our results demonstrated that elevated TGF- $\beta 1$ and decreased MMP9 were associated with the activation of Nrf2 in HB-mediated wound healing.

Taken together, our findings demonstrated activation of Nrf2 by HB liniment accelerated diabetic wound healing. This study provided experiment evidence for topical HB liniment treatment for diabetic wounds and highlighting the effect of activating Nrf2 in diabetic wound healing. However, we only focused on the effect of $\mathrm{HB}$ on oxidative damage, whether it had an effect on inflammation, angiogenesis and antibiosis remained further investigation. In addition, the components in $\mathrm{HB}$ liniment which really played a role in activating Nrf2 need to be clarified in the future study.

\section{Conclusions}

In summary, an obvious protective effect of $\mathrm{HB}$ treatment against diabetic wound healing was observed, and reduction 
in oxidative stress may play a vital role in HB-mediated wound healing. RNA-seq analysis and further experiment suggested that the activation of $\mathrm{Nrf} 2$ and its downstream antioxidant genes reduced apoptosis and oxidative damage, thereby improving HB-mediated wound healing. And 54 compounds in $\mathrm{HB}$ liniment were identified to have an effect on $\mathrm{Nfe} 2 \mathrm{l} 2$ and its downstream genes, which had a synergistically inhibitive effect on oxidative stress. In addition, HB liniment, increased TGF- $\beta 1$ level, and reduced MMP9, thereby contributing to the acceleration of diabetic wound healing. Our findings offered experimental evidence of $\mathrm{HB}$ liniment in the treatment of diabetic wounds and also improved our understanding of diabetic wound healing, supporting development of Nrf2-based therapy in the treatment of diabetic ulcer.

\section{Data Availability}

The data about the RNA-seq analysis was uploaded into NCBI https://www.ncbi.nlm.nih.gov/sra/PRJNA532974 (SRP193129) which can be downloaded. As for the other data used to support the findings of this study are available from the corresponding author upon request.

\section{Conflicts of Interest}

The authors declare that they have no conflicts of interest.

\section{Authors' Contributions}

Jingjing Zhang and Rui Zhou contributed equally to this work.

\section{Acknowledgments}

The authors would like to acknowledge the financial support from National Key R\&D Plan (no. 2017YFC1702605), National Natural Science Foundation of China (no. 81974550), and Fundamental Research Funds for the Central Universities (no. ZZ13-YQ-046). The author wound like to thank Xin Li for the assistant in the network analysis. The authors disclose that this project was partially funded by Shandong Hanfang Pharmaceutical Co., Ltd, who also provided Huangbai liniment (Chinese medicine character: Z10950097) for this research. The authors promisse that the authenticity and conclusions of this study will not be affected by the funding.

\section{Supplementary Materials}

Table S1. The RNA-seq data of nondiabetic wound (Con), STZ-induced diabetic wound with or without HB treatment. The gene expressions of different ss were expressed as FPKM and the fold change and false discovery rate (FDR). (Supplementary Materials)

\section{References}

[1] V. R. Driver, M. Fabbi, L. A. Lavery, and G. Gibbons, "The costs of diabetic foot: the economic case for the limb salvage team," Journal of Vascular Surgery, vol. 52, no. 3, pp. 17S22S, 2010.

[2] G. E. Reiber, B. A. Lipsky, and G. W. Gibbons, "The burden of diabetic foot ulcers," American Journal of Surgery, vol. 176, no. 2, pp. 5S-10S, 1998.

[3] R. Blakytny and E. B. Jude, "Altered molecular mechanisms of diabetic foot ulcers," The International Journal of Lower Extremity Wounds, vol. 8, no. 2, pp. 95-104, 2009.

[4] V. Falanga, "Wound healing and its impairment in the diabetic foot," Lancet, vol. 366, no. 9498, pp. 1736-1743, 2005.

[5] C. Bianchi and S. Del Prato, "Metabolic memory and individual treatment aims in type 2 diabetes-outcome-lessons learned from large clinical trials," The Review of Diabetic Studies, vol. 8, no. 3, pp. 432-440, 2011.

[6] A. K. Arya, R. Tripathi, S. Kumar, and K. Tripathi, "Recent advances on the association of apoptosis in chronic non healing diabetic wound," World Journal of Diabetes, vol. 5, no. 6, pp. 756-762, 2014.

[7] M. Cano Sanchez, S. Lancel, E. Boulanger, and R. Neviere, "Targeting oxidative stress and mitochondrial dysfunction in the treatment of impaired wound healing: a systematic review," Antioxidants, vol. 7, no. 8, p. 98, 2018.

[8] Z. Zhang, Z. Zi, E. E. Lee et al., "Differential glucose requirement in skin homeostasis and injury identifies a therapeutic target for psoriasis," Nature Medicine, vol. 24, no. 5, pp. 617$627,2018$.

[9] F. P. Beserra, A. J. Vieira, L. F. S. Gushiken et al., "Lupeol, a dietary Triterpene, enhances wound healing in streptozotocininduced hyperglycemic rats with modulatory effects on inflammation, oxidative Stress, and angiogenesis," Oxidative Medicine and Cellular Longevity, vol. 2019, Article ID 3182627, 20 pages, 2019.

[10] T. He, T. Quan, Y. Shao, J. J. Voorhees, and G. J. Fisher, "Oxidative exposure impairs TGF- $\beta$ pathway via reduction of type II receptor and SMAD3 in human skin fibroblasts," Age, vol. 36, no. 3, p. 9623, 2014.

[11] M. J. Davies, "The oxidative environment and protein damage," Biochimica et Biophysica Acta, vol. 1703, no. 2, pp. 93-109, 2005.

[12] A. E. Loo, Y. T. Wong, R. Ho et al., "Effects of hydrogen peroxide on wound healing in mice in relation to oxidative damage," PLoS One, vol. 7, no. 11, 2012.

[13] B. Hantke, C. Lahmann, K. Venzke et al., "Influence of flavonoids and vitamins on the MMP- and TIMP-expression of human dermal fibroblasts after UVA irradiation," Photochemical \& Photobiological Sciences, vol. 1, no. 10, pp. 826-833, 2002.

[14] L. Wang, G. B. Zhou, P. Liu et al., "Dissection of mechanisms of Chinese medicinal formula Realgar-Indigo naturalis as an effective treatment for promyelocytic leukemia," Proceedings of the National Academy of Sciences of the United States of America, vol. 105, no. 12, pp. 4826-4831, 2008.

[15] J. Zhang, F. Guo, J. Wei et al., “An integrated approach to identify critical transcription factors in the protection against hydrogen peroxide-induced oxidative stress by Danhong injection," Free Radical Biology \& Medicine, vol. 112, pp. 480-493, 2017.

[16] X. L. Tong, L. Dong, L. Chen, and Z. Zhen, “Treatment of diabetes using traditional Chinese medicine: past, present and future," The American Journal of Chinese Medicine, vol. 40, no. 5, pp. 877-886, 2012. 
[17] Committee, CP, China Pharmacopoeia, vol. 1, Chemical Industry Press, 2015.

[18] X. Hou, X. Jun, P. Wang et al., "Analysis on clinical efficacy of compound Huangbai fluid as adjunctive treatment for diabetic foot ulcer," Chinese Journal of Experimental Traditional Medical Formulae, vol. 22, no. 4, pp. 159-163, 2016.

[19] L. You-shan, Z. Qi, and Y. Bo-hua, "Efficacy and safety in a multi-center clinical trial for analyzing compound fluid of cortex phellodendri in the external treatment of diabetic foot ulcers," Chinese Journal of New Drugs, vol. 25, no. 20, pp. 2344-2348, 2016.

[20] L. You-shan and Y. Bo-hua, "Effects of compound fluid of cortex phellodendri on inflammatory cytokines and growth factors in external treatment of diabetic foot ulcer," Chinese Journal of New Drugs, vol. 23, no. 10, pp. 1163-1166, 2014.

[21] H. Zheng, S. A. Whitman, W. Wu et al., "Therapeutic potential of Nrf2 activators in streptozotocin-induced diabetic nephropathy," Diabetes, vol. 60, no. 11, pp. 3055-3066, 2011.

[22] P. Shannon, A. Markiel, O. Ozier et al., "Cytoscape: a software environment for integrated models of biomolecular interaction networks," Genome Research, vol. 13, no. 11, pp. 24982504, 2003.

[23] N. T. Moldogazieva, I. M. Mokhosoev, T. I. Mel'nikova, Y. B. Porozov, and A. A. Terentiev, "Oxidative Stress and advanced lipoxidation and glycation end products (ALEs and AGEs) in aging and age-related diseases," Oxidative Medicine and Cellular Longevity, vol. 2019, Article ID 3085756, 14 pages, 2019.

[24] J. D. Hayes and A. T. Dinkova-Kostova, "The Nrf2 regulatory network provides an interface between redox and intermediary metabolism," Trends in Biochemical Sciences, vol. 39, no. 4, pp. 199-218, 2014.

[25] M. Long, M. Rojo de la Vega, Q. Wen et al., "An essential role of NRF2 in diabetic wound healing," Diabetes, vol. 65, no. 3, pp. 780-793, 2016.

[26] M. A. Soares, O. D. Cohen, Y. C. Low et al., "Restoration of Nrf2 signaling normalizes the regenerative niche," Diabetes, vol. 65, no. 3, pp. 633-646, 2016.

[27] L. G. Higgins, M. O. Kelleher, I. M. Eggleston, K. Itoh, M. Yamamoto, and J. D. Hayes, "Transcription factor Nrf2 mediates an adaptive response to sulforaphane that protects fibroblasts in vitro against the cytotoxic effects of electrophiles, peroxides and redox-cycling agents," Toxicology and Applied Pharmacology, vol. 237, no. 3, pp. 267-280, 2009.

[28] J. Lu and A. Holmgren, "The thioredoxin antioxidant system," Free Radical Biology \& Medicine, vol. 66, pp. 75-87, 2014.

[29] K. C. Wu, J. Y. Cui, and C. D. Klaassen, "Beneficial role of Nrf2 in regulating NADPH generation and consumption," Toxicological Sciences, vol. 123, no. 2, pp. 590-600, 2011.

[30] E. Pontiki and D. Hadjipavlou-Litina, "Multi-target cinnamic acids for oxidative stress and inflammation: design, synthesis, biological evaluation and modeling studies," Molecules, vol. 24, no. 1, p. 12, 2018.

[31] G. Wang, Z. Lei, Q. Zhong et al., "Enrichment of caffeic acid in peanut sprouts and evaluation of its in vitro effectiveness against oxidative stress-induced erythrocyte hemolysis," Food Chemistry, vol. 217, pp. 332-341, 2017.

[32] T. M. Hung, J. P. Lee, B. S. Min et al., "Magnoflorine from Coptidis Rhizoma protects high density lipoprotein during oxidant stress," Biological \& Pharmaceutical Bulletin, vol. 30, no. 6, pp. 1157-1160, 2007.
[33] F. Guglielmi, C. Luceri, L. Giovannelli, P. Dolara, and M. Lodovici, "Effect of 4-coumaric and 3,4-dihydroxybenzoic acid on oxidative DNA damage in rat colonic mucosa," The British Journal of Nutrition, vol. 89, no. 5, pp. 581-587, 2003.

[34] T. Luo, X. Y. Shen, S. Li, T. Ouyang, Q. A. Mai, and H. Q. Wang, "The protective effect of jatrorrhizine against oxidative stress in primary rat cortical neurons," CNS \& Neurological Disorders Drug Targets, vol. 16, no. 5, pp. 617-623, 2017.

[35] Y. Zhang, F. Feng, T. Chen, Z. Li, and Q. W. Shen, "Antidiabetic and antihyperlipidemic activities of forsythia suspensa (Thunb.) vahl (fruit) in streptozotocin-induced diabetes mice," Journal of Ethnopharmacology, vol. 192, pp. 256-263, 2016.

[36] J. Roslan, N. Giribabu, K. Karim, and N. Salleh, "Quercetin ameliorates oxidative stress, inflammation and apoptosis in the heart of streptozotocin-nicotinamide-induced adult male diabetic rats," Biomedicine \& Pharmacotherapy, vol. 86, pp. 570-582, 2017.

[37] K. Vinothiya and N. Ashokkumar, "Modulatory effect of vanillic acid on antioxidant status in high fat diet- induced changes in diabetic hypertensive rats," Biomedicine \& Pharmacotherapy, vol. 87, pp. 640-652, 2017.

[38] S. Bae, K. Lim, H. Cha et al., "Arctiin blocks hydrogen peroxide-induced senescence and cell death though microRNA expression changes in human dermal papilla cells," Biological Research, vol. 47, no. 1, p. 50, 2014.

[39] J. Marhuenda, S. Medina, P. Martínez-Hernández et al., "Melatonin and hydroxytyrosol protect against oxidative stress related to the central nervous system after the ingestion of three types of wine by healthy volunteers," Food \& Function, vol. 8, no. 1, pp. 64-74, 2017.

[40] C. Guo, S. Wang, J. Duan et al., "Protocatechualdehyde protects against cerebral ischemia-reperfusion-induced oxidative injury via protein kinase $\mathrm{C} \varepsilon / \mathrm{Nrf} 2 / \mathrm{HO}-1$ pathway," Molecular Neurobiology, vol. 54, no. 2, pp. 833-845, 2017.

[41] F. E. Wirngo, M. N. Lambert, and P. B. Jeppesen, "The physiological effects of dandelion (Taraxacum officinale) in type 2 diabetes," The Review of Diabetic Studies, vol. 13, no. 2-3, pp. 113-131, 2016.

[42] M. H. Han, C. Park, D. S. Lee et al., "Cytoprotective effects of esculetin against oxidative stress are associated with the upregulation of Nrf2-mediated NQO1 expression via the activation of the ERK pathway," International Journal of Molecular Medicine, vol. 39, no. 2, pp. 380-386, 2017.

[43] P. Stanely Mainzen Prince and A. J. Roy, “_p_- -Coumaric acid attenuates apoptosis in isoproterenol-induced myocardial infarcted rats by inhibiting oxidative stress," International Journal of Cardiology, vol. 168, no. 4, pp. 3259-3266, 2013.

[44] C. Chaowuttikul, C. Palanuvej, and N. Ruangrungsi, "Pharmacognostic Specification, Chlorogenic Acid Content, and In vitro Antioxidant Activities of Lonicera japonica Flowering Bud," Pharmacognosy Research, vol. 9, no. 2, pp. 128-132, 2017.

[45] S. Kim, Y. W. Chin, and J. Cho, "Protection of cultured cortical neurons by luteolin against oxidative damage through inhibition of apoptosis and induction of heme oxygenase-1," Biological \& Pharmaceutical Bulletin, vol. 40, no. 3, pp. 256-265, 2017.

[46] Y. Ren, P. Houghton, and R. C. Hider, "Relevant activities of extracts and constituents of animals used in traditional Chinese medicine for central nervous system effects 
associated with Alzheimer's disease," The Journal of Pharmacy and Pharmacology, vol. 58, no. 7, pp. 989-996, 2006.

[47] N. Polera, M. Badolato, F. Perri, G. Carullo, and F. Aiello, "Quercetin and its natural sources in wound healing management," Current Medicinal Chemistry, vol. 26, no. 31, pp. 58255848, 2019.

[48] S. Wang, J. Yao, B. Zhou et al., "Bacteriostatic effect of quercetin as antibiotic alternative in vivo and its antibacterial mechanism in vitro," Journal of Food Protection, vol. 81, no. 1, pp. 68-78, 2018.

[49] O. M. Ahmed, T. Mohamed, H. Moustafa, H. Hamdy, R. R. Ahmed, and E. Aboud, "Quercetin and low level laser therapy promote wound healing process in diabetic rats _via_structural reorganization and modulatory effects on inflammation and oxidative stress," Biomedicine \& Pharmacotherapy, vol. 101, pp. 58-73, 2018.

[50] E. B. Jude, R. Blakytny, J. Bulmer, A. J. Boulton, and M. W. Ferguson, "Transforming growth factor-beta 1, 2, 3 and receptor type I and II in diabetic foot ulcers," Diabetic Medicine, vol. 19, no. 6, pp. 440-447, 2002.

[51] Y. Liu, D. Min, T. Bolton et al., "Increased matrix metalloproteinase-9 predicts poor wound healing in diabetic foot ulcers," Diabetes Care, vol. 32, no. 1, pp. 117-119, 2009.

[52] T. Dinh, F. Tecilazich, A. Kafanas et al., "Mechanisms involved in the development and healing of diabetic foot ulceration," Diabetes, vol. 61, no. 11, pp. 2937-2947, 2012.

[53] E. P. Amento and L. S. Beck, "TGF-beta and wound healing," Ciba Foundation Symposium, vol. 157, pp. 115-123, 1991.

[54] S. M. Ayuk, H. Abrahamse, and N. N. Houreld, "The role of matrix metalloproteinases in diabetic wound healing in relation to photobiomodulation," Journal Diabetes Research, vol. 2016, article 2897656, 9 pages, 2016.

[55] M. Gao, T. T. Nguyen, M. A. Suckow et al., "Acceleration of diabetic wound healing using a novel protease-anti-protease combination therapy," Proceedings of the National Academy of Sciences of the United States of America, vol. 112, no. 49, pp. 15226-15231, 2015. 